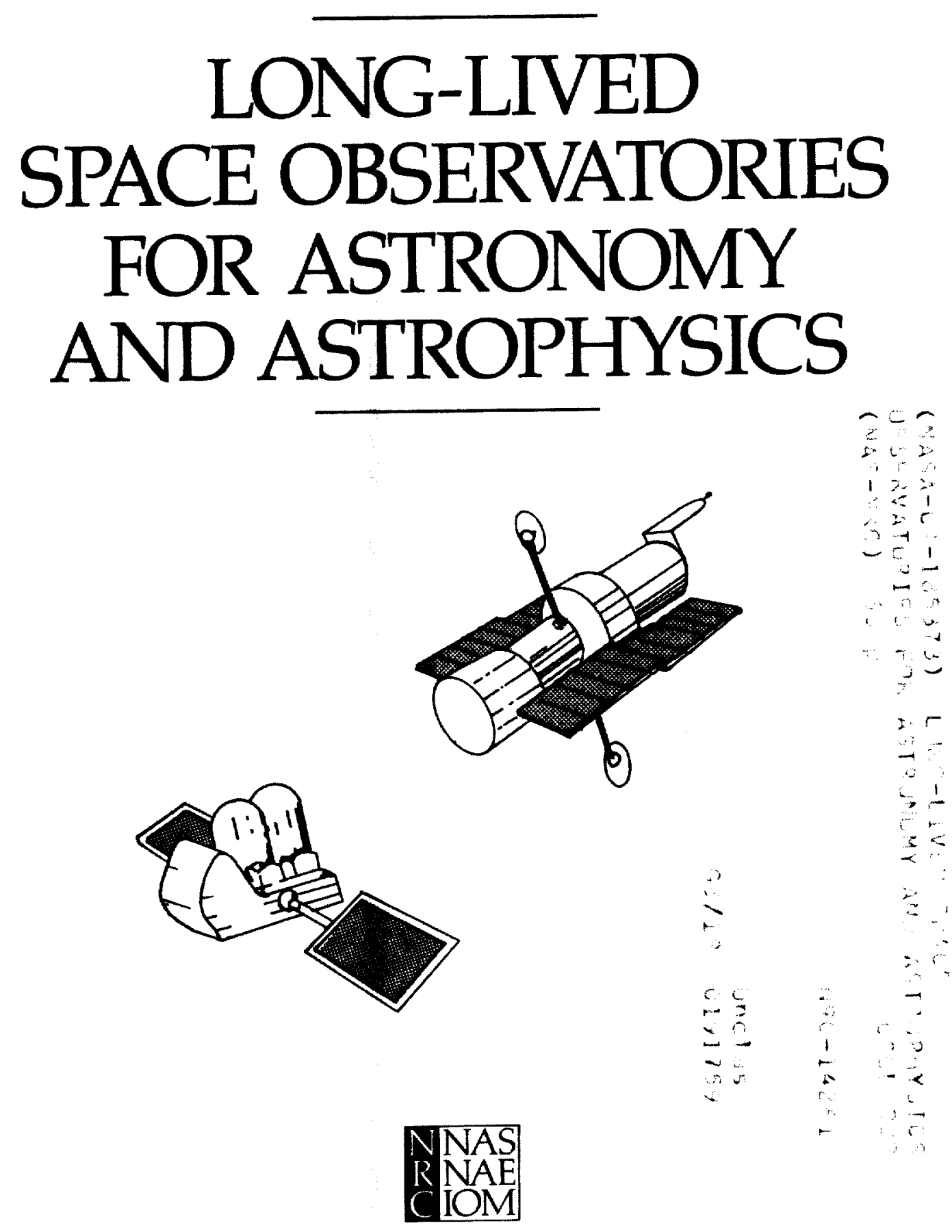




\section{LONG-LIVED SPACE OBSERVATORIES FOR ASTRONOMY AND ASTROPHYSICS}

Committee on Space Astronomy and Astrophysics

Space Science Board

Commission on Physical Sciences, Mathematics, and Resources

National Research Council 
NOTICE: The project that is the subject of this report was approved by the Governing Doard of the National Research Council, whose members are drawn from the councils of the National Academy of Sciences, the National Academy of Engineering, and the Institute of Medicine. The members of the committee responsible for the report were chosen for their special competences and with regard for appropriate balance.

This report has been reviewed by a group other than the authors according to procedures approved by a Report Review Committee consisting of members of the National Academy of Sciences, the National Academy of Engineering, and the Institute of Medicine.

The National Academy of Sciences is a private, nonprofit, self-perpetuating socjety of distinguished scholars engaged in scientific and engineering research, dedicated to the furtherance of science and technology and to their use for the general welfare. Upon the authority of the charter granted to it by the Congress in 1863 , the Academy has a mandate that requires it to advise the federal government on scientific and technical matters. Dr. Frank Press is president of the National Academy of Sciences.

The National Academy of Engineering was established in 1964, under the charter of the National Academy of Sciences, as a parallel organization of outstanding engineers. It is autonomous in its administration and in the selection of its members, sharing with the National Academy of Sciences the responsibility for advising the federal government. The National Academy of Engineering also sponsors engineering programs aimed at meeting national needs, encourages education and research, and recognizes the superior achievernents of engineers. Dr. Robert $M$. White is president of the National Academy of Engineering.

The Institute of Medicine was established in 1970 by the National Academy of Sciences to secure the services of eminent members of appropriate professions in the examination of policy matters pertaining to the health of the public. The Institute acts under the responsibility given to the National Academy of Sciences by its congressional charter to be an adviser to the federal government and, upon its own initiative, to identify issues of medical care, research, education. Dr. Samuel O. Thier is president of the Institute of Medicine.

The National Research Council was organized by the National Academy of Sciences in 1916 to associate the broad community of science and technology with the Academy's purposes of furthering knowledge and advising the federal government. Functioning in accordance with general policies determined by the Academy, the Council has become the principal operating agency of both the National Academy of Sciences and the National Academy of Engineering in providing services to the government, the public, and the scientific and engineering communities. The Council is administered jointly by both Academies and the Institute of Medicine. Dr. Frank Press and Dr. Robert M. White are chairman and vice chairman, respectively, of the National Research Council.

Support for this project was provided by Contract NASW 3482 between the National Academy of Sciences and the National Aeronautics and Space Administration.

Available from

Space Science Bonrd

National Research Council

2101 Constitution Ave., N.W.

Washington, D.C. 20418

Printed in the United States of America 


\section{COMMITTEE ON SPACE ASTRONOMY AND ASTROPHYSICS}

Blair D. Savage, University of Wisconsin, Chairman Eric E. Becklin, University for Astronomy Steven V.W. Beckwith, Cornell University Lennox L. Cowie, University of Hawaii Andrea K. Dupree, Center for Astrophysics James L. Elliot, Massachusetts Institute of Technology John S. Gallagher, Lowell Observatory David J. Helfand, Columbia University Edward F. Jenkins, Princeton University Kenneth J. Johnston, Naval Research Laboratory Michael Jura, University of California at Los Angeles James Kurfess, Naval Research Laboratory Steven S. Murray, Center for Astrophysics Craig L. Sarazin, University of Virginia Roger Ulrich, University of California at Los Angeles

Mark E. Wiedenbeck, Enrico Fermi Institute, University of Chicago

Richard C. Hart, Executive Secretary 


\section{SPACE SCIENCE BOARD}

Thomas M. Donahue, University of Michigan, Chairman D. James Baker, Joint Oceanographic Institutions, Inc. Roger D. Blandford, California Institute of Technology Larry Esposito, University of Colorado Lennard Fisk, University of New Hampshire Jonathan Grindlay, Center for Astrophysics Donald Hall, University of Hawaii

William Kaula, NOAA

Harold Klein, The University of Santa Clara John Leibacher, National Solar Observatory Robert Pepin, University of Minnesota Roger Phillips, Southern Methodist University David Raup, University of Chicago Christopher Russell, University of California, Los Angeles Blair D. Savage, University of Wisconsin

John Simpson, Enrico Fermi Institute, University of Chicago George L. Siscoe, University of California, Los Angeles L. Dennis Smith, Purdue University Darrell Strobel, Johns Hopkins University

Dean P. Kastel, Staff Director

Richard C. Hart, Senior Program Officer 


\section{COMMISSION ON PHYSICAL SCIENCES, MATHEMATICS, AND RESOURCES}

Norman Hackerman, Robert A. Welch Foundation, Chairman George F. Carrier, Harvard University

Dean E. Eastman, IBM, T. J. Watson Research Center

Marye Anne Fox, University of Texas

Gerhart Friedlander, Brookhaven National Laboratory

Lawrence W. Funkhouser, Chevron Corporation (retired)

Phillip A. Griffiths, Duke University

J. Ross Macdonald, University of North Carolina, Chapel Hill

Charles J. Mankin, Oklahoma Geological Survey

Perry L. McCarty, Stanford University

Jack E. Oliver, Cornell University

Jeremiah P. Ostriker, Princeton University Observatory

William D. Phillips, Mallinckrodt, Inc.

Denis J. Prager, MacArthur Foundation

David M. Raup, University of Chicago

Richard J. Reed, University of Washington

Robert E. Sievers, University of Colorado

Larry L. Smarr, National Center for Supercomputing Applications

Edward C. Stone, Jr., California Institute of Technology

Karl K. Turekian, Yale University

George W. Wetherill, Carnegie Institution of Washington

Irving Wladawsky-Berger, IBM Corporation

Raphael G. Kasper, Executive Director

Lawrence E. McCray, Associate Executive Director 



\section{Preface}

\section{PRECEDING PAGE ELAIK NOT FILMED}

This report discusses NASA's plan to build and launch a fleet of long-lived space observatories that includes the Hubble Space Telescope (HST), the Gamma Ray Observatory (GRO), the Advanced X-Ray Astrophysics Observatory (AXAF), and the Space Infrared Telescope Facility (SIRTF). These facilities are expected to have a profound impact on the sciences of astronomy and astrophysics. The long-lived observatories will provide new insights about astronomical and astrophysical problems that range from the presence of planets orbiting nearby stars to the large-scale distribution and evolution of matter in the universe.

Because of concern over the future of the program and the large costs associated with operating and maintaining major observatories in space, the Committee on Space Astronomy and Astrophysics (CSAA) under the chairmanship of Jacques M. Beckers began a study in November 1984 of NASA's long-lived observatory program, often referred to as the "Great Observatory" program. The CSAA Long-Lived Observatories Study was chaired by Michael Jura and was charged with addressing the following points:

- The scientific rationale for long-lived observatories in space.

- The need for space facilities covering a wide wavelength range.

- A balanced program for space observatories.

vii

PAES UL_ INTENTIONALIX BLANK 
- The relation of space observatories to ground-based observations.

- Management and operation policies for long-lived space observatories.

- Maintenance and refurbishment strategies.

- The sociology of funding and manpower arrangements.

In preparing this report, the CSAA met approximately six times over a two and a half year period. The committee consulted many members of the space astronomy and astrophysics community and listened to presentations by scientists, project managers, and engineers involved with major space missions and large ground-based observatories. The committee also carefully reviewed the many existing documents on the subject of space observatories. The charge to the committee was enormous in scope, and during the study it became clear that no single study will be able to adequately address the large number of very difficult technical, operational, and management issues involving a fleet of long-lived observatories in space. We hope, however, that the report we have created is a good first step toward identifying many of the issues and possibly solving some of them. Clearly much more work will be needed in the future.

Our report was in very rough draft form when the Space Shuttle Challenger exploded on January 28, 1986. It has taken nearly two years for the nation and NASA to begin to realize the full implications of that event. Our report has been written with the assumption that our nation's space program (both manned and unmanned) will recover from the disaster and will eventually return to a period of great discovery such as we had in the late 1960s and early 1970s. A space program that aspires to a position of international leadership must be strong in the astronomical and astrophysical sciences. Embarking on the long-lived observatory program will help the United States to achieve that leadership position. However, NASA must make special efforts to ensure that such a large program as the Great Observatory program is not undertaken at the expense of other important but smaller programs such as the exceedingly successful Explorer program (see the CSAA report The Explorer Program for Astronomy and Astrophysics, National Academy Press, 1986). To make such a balanced space astronomy program possible, it will be necessary for NASA to develop an operational plan for the Great Observatories that minimizes the operational and refurbishment costs for the entire suite of observatories. 
This report was produced with the help of many of our scientific, technical, and management colleagues. Although we cannot acknowledge them all individually, we are very grateful for their many thoughtful contributions. We especially want to acknowledge the help received from NASA headquarters and in particular from C. Pellerin, A. Bunner, E. Weiler, and L. Peterson. We also wish to thank the executive secretary of the CSAA, Richard C. Hart, who was extremely important in providing encouragement, technical support, and many of the organizational ideas that made our basic study and the writing of this report possible.

Michael Jura, Chairman

CSAA Long-Lived Space Observatories Study

Blair D. Savage, Chairman

Committee on Space Astronomy and Astrophysics 
- 


\section{Contents}

1. EXECUTIVE SUMMARY 1

2. SCIENTIFIC RATIONALE FOR LONG-LIVED OBSERVATORIES IN SPACE

3. PREVIOUS STUDIES 21

4. REQUIREMENTS FOR A LONG-LIVED
OBSERVATORY PROGRAM

APPENDIX: ABBREVIATIONS USED IN TEXT 49

\section{PRECEDIING PAGE ELAINK NOT FILMED}

$\mathbf{x i}$

PAGE $X$ INIENTIONALLY BLANK 


\section{1 \\ Executive Summary}

The National Aeronautics and Space Administration (NASA) is currently planning a long-lived observatory program (often called the "Great Observatory" program) that includes the Hubble Space Telescope (HST), the Gamma Ray Observatory (GRO), the Advanced X-Ray Astrophysics Facility (AXAF), and the Space Infrared Telescope Facility (SIRTF). This combination of facilities will make possible high-sensitivity observations over 13 decades in wavelength ranging from $10^{-14} \mathrm{~cm}$ to $7 \times 10^{-2} \mathrm{~cm}\left(10^{-6} \AA\right.$ to $700 \mu \mathrm{m})$. HST will observe deep into the universe in visible and ultraviolet wavelengths, expanding the volume of observable space several hundred times. GRO will explore the most energetic part of the electromagnetic spectrum. AXAF will cover the X-ray portion of the spectrum with a hundredfold improvement in sensitivity over previous satellites. SIRTF will span the infrared with a thousandfold improvement in sensitivity over IRAS. With these high sensitivities and the extensive wavelength coverage, the Great Observatories will profoundly affect the science of astronomy and astrophysics. The Great Observatories will provide new insights about topics ranging from the presence of planets orbiting nearby stars, to the nature of the mysterious energy sources in galactic nuclei, and to the large-scale distribution and evolution of matter in the universe. 
An important concern to NASA and the scientific community is the operation and maintenance cost of the four observatories described above. The HST cost about $\$ 1.3$ billion (1984 dollars) to build and is estimated to require $\$ 160$ million (1986 dollars) a year to operate and maintain. If HST is operated for 20 years, the accumulated costs will be considerably more than those required for its construction. Therefore, it is essential to plan carefully for observatory operations and maintenance before a long-lived facility is constructed. The primary goal of this report is to help NASA develop guidelines for the operations and management of these future observatories so as to achieve the best possible scientific results for the resources available. Although the Shuttle disaster has caused serious disruptions in the plans for space astrophysics, building observatories in space deserves the highest possible priority.

\section{RECOMMENDATIONS}

1. We recommend that $H S T$ and GRO be given high priority for launch with the Space Shuttle. These unique observatories will challenge the fundamental scientific questions of our time and help begin to restore our nation's confidence in the U.S. space program.

2. We recommend that NASA establish plans, including a guest investigator program, to extend the mission lifetime of the $G R O$ beyond the currently designed 2 to 9 years.

3. We concur with the recommendations of the Astronomy Survey Committee Report (1980), which urges the construction of $A X A F$ and $S I R T F$. These two missions will play a fundamental role in the future progress of astronomy and astrophysics.

4. We recommend that $N A S A$ vigorously plan to minimize the operational and refurbishment costs of the entire suite of long-lived space observatories. The instrumentation and operating modes of AXAF and SIRTF should be as simple as possible, consistent with the scientific goals of each observatory. Options for undertaking AXAF and SIRTF without manned intervention as well as with minimal requirements for manned space activities should be thoroughly investigated.

5. We recommend the active involvement of the broad community of users in all phases of the long-lived observatory program in order to foster the most imaginative and fruitful utilization of these facilities. A committee of distinguished astronomers reporting to

\section{ORIGINAL PAGE IS OF POOR QUALITY}


NASA should be established to provide oversight to the entire program of long-lived astronomical and astrophysical observatories in space. This committee should intercompare the scientific productivity of the various facilities and should advise NASA on important decisions involving these facilities. In order to minimize costs, this committee should make recommendations concerning the distribution of funding among the various elements of the overall program. Proper consideration of the appropriate tradeoffs must include clearly established criteria for the phase-down of each element of the program.

6. We recommend that each long-lived observatory have a dedicated science operations staff with a well-defined scope of responsibilities in order that each observatory can be utilized to its full potential. The observatory staffs must be intimately familiar with the mission planning, instrument capabilities, operating modes, and data reduction schemes. Characteristics of the science operations activities to ensure efficient operations at minimal cost are as follows: (a) A core operations team that is assembled contemporaneously with the decision to commit to the construction of the observatory. There are many advantages to considerable decentralization of scientific activities that are not real-time critical; it may not be necessary to locate all aspects of science operations at a distinct "Institute." (b) Competitive selection with peer review at all appropriate phases. This includes the detailed implementation plan, the institution or consortium responsible for that implementation, and the individuals who serve in key scientific positions.

7. We recommend that international cooperation be encouraged in the long-lived observatory program. This cooperation should enhance the scientific productivity of the observatories and help reduce costs to NASA. Full participation of foreign partners in the sharing of the observatory maintenance and operations cost must be encouraged.

8. We recommend that the astronomical community establish and implement detailed plans for providing complementary and supporting ground-based observations to the measurements expected from the Great Observatories. These plans should include a strategy for the archiving and distribution of visible, infrared, and radio data obtained from the ground-based instruments. 


\section{2 \\ Scientific Rationale for Long-Lived \\ Observatories in Space}

In the years since the organization of NASA in 1958, astronomy has been completely revolutionized. For centuries limited to observations in the visible band of the electromagnetic spectrum, astronomy has now used virtually all wavelengths from about $10^{-13} \mathrm{~cm}$ (corresponding to gamma rays of energy $1 \mathrm{GeV}$ ) to about $3 \times 10^{6} \mathrm{~cm}$ (the cutoff at radio wavelengths imposed by interplanetary plasma). Starting with its charter "to contribute materially to the expansion of human knowledge of phenomena in the atmosphere and space," NASA has played a leading role in this revolution, by flying detectors above the atmosphere to study gamma rays, X-rays, ultraviolet, visible, infrared radiation, and radio emission.

We now know that the visible universe is only a tiny part of reality. Each spectral band reveals phenomena totally unexpected from studies of visible radiation-some so important that they have definitively changed our understanding of the universe. In the radio band we learned for the first time of quasars, whose enormous luminosities seem to require a radically new energy source-such as accretion onto black holes - that was completely unsuspected from optical studies. And the cosmic microwave background discovered in 1965 helped to reveal the physical laws that rule the universe as a whole. Gamma-ray bursters may be neutron stars, a new 
state of matter predicted theoretically, but studied in detail for the first time by radio and X-ray astronomers. All of the cases in which we suspect the presence of that enigmatic object-a stellar black hole-were discovered by X-ray astronomers. Ultraviolet astronomy revealed that our galaxy is filled with superhot gas. Infrared studies have for the first time revealed stars with disks of dust in orbit around them, much like that from which the solar system formed, as well as galaxies that radiate unprecedented power almost entirely in the infrared.

\section{THE GOALS OF ASTRONOMY AND ASTROPHYSICS}

Some of the major contemporary goals of astronomy and astrophysics include (1) understanding the formation of planets and of the solar system, including the conditions for life to form and evolve in the universe; (2) studying the formation of stars and their subsequent evolution; (3) probing the earliest stages of the universe to learn how galaxies form and evolve to their current state; and (4) learning more about cosmic energy sources, including active galaxies and quasars.

Astronomy, along with physics, is also charged with understanding the laws that govern the universe. Several times in history, astronomy has played a key role in revealing these laws, as when Newton discovered the laws of gravitation and of motion and tested them against the motions of the planets.

At present, we appear to be standing on the threshold of an extraordinary synthesis in which the four forces of nature many be theoretically explained as manifestations of but a single unified force. The current work of particle physicists in this area has powerful implications for the origin of matter in the universe and its distribution in space-implications that can be tested by astronomical measurements. In our lifetimes we may see a dramatic reenactment of Newton's discoveries, in which profound insights into the nature of matter stemming from the most abstract branches of theoretical physics are validated by astronomical observations.

\section{THE POWER OF SPACE ASTRONOMY}

A simple law of physics governs all astronomical observations from the ground or from space: the hotter the object, the shorter the wavelength of the bulk of the emitted radiation. In retrospect 
we can see how it is that the true nature of the universe was hidden from optical astronomers. Visible wavelengths are readily emitted by bodies ranging in temperature from about 3,000 to $30,000 \mathrm{~K}$-the temperature range for most stars. Cooler bodies, such as cool stars, planets, and interstellar clouds, emit largely in the infrared or even radio bands, while hotter bodies, such as collapsed stars, hot gases around stars and in space, and explosions of stars or of galaxies are best observed in the ultraviolet, X-ray, or even gamma-ray bands. Rarified regions containing relativistic particles shine brightly at radio and $\mathrm{X}$-ray wavelengths but are nearly invisible with optical telescopes. The lesson is clear-full wavelength coverage is essential if astrophysicists are to build models of phenomena that stand up to further tests.

The astronomer's need to observe at various wavelengths is frustrated by the earth's atmosphere that blocks virtually all of the infrared, ultraviolet, X-ray, and gamma-ray bands. Even in the visible part of the spectrum, the rays that do penetrate the atmosphere are refracted significantly, distorting astronomical images and destroying critically important information. In this situation, the advent of space astronomy created a revolution in the field of astronomy and astrophysics. By opening up the hidden decades of the electromagnetic spectrum, completely new facets of the universe have been revealed.

NASA has wisely proceeded in an orderly fashion to study each region of the spectrum. As a first step, rocket and balloon payloads have been used to verify the existence of some astronomical sources at the chosen wavelength. A survey has been conducted to give positions of sources for further study, and specialized instruments have been flown to study specific aspects of the brighter sources. Finally, major space observatories that have large collecting areas and comprehensive complements of instruments are required. Through long-term pointed observations, these observatories can conduct penetrating studies of a variety of objects, providing the data to discover what is really going on. In 1986, except for the extreme ultraviolet range to be explored by EUVE in the early 1990s and the submillimeter range yet to be explored, the initial surveys are largely complete and astronomy is ready for long-lived observatories in space. 


\section{THE LONG-LIVED OBSERVATORY PROGRAM}

A full understanding of any astronomical phenomena requires several cycles of observation and measurement: data analysis and interpretation; the construction of theoretical hypotheses and detailed models; and the development of tests and predictions of the models. In addition, a new kind of astrophysical object may be detected first in one part of the spectrum, but the understanding of these objects may require observations in completely different regions of the spectrum. Often, the strongest predictions of a theory that explains the emission in one wavelength regime concern the radiation in another regime. For example, quasars were first detected as radio sources, but their identification with extremely distant objects was based on the redshift of their optical line emission. Present theories for this optical line emission are based on ionization and heating models involving X-rays and ultraviolet light, and can be tested by observations at these wavelengths. The X-ray emission itself may be due to an inverse Compton process; this hypothesis makes several strong predictions regarding the infrared emission of these objects.

As a second example, consider the enigmatic galactic object SS 433, which may be a miniature version of a quasar. This object was first noted as a slightly unusual optical star, but was not studied extensively until it was discovered to be a very luminous and violently variable source of both X-rays and radio. Optical astronomers then reexamined the star and found evidence that it was ejecting powerful beams of gas in opposite directions at a quarter the speed of light. Radio observations of the region of our galaxy around SS 433 then showed that these beams had blasted a very large, elongated hole (named W 50) in the surrounding gas. The beams themselves were first imaged by radio and then X-ray observations. Recently, measurement of the X-ray spectrum and temporal variation of this source have shown that the beams are eclipsed by an accretion disk surrounding a compact object at the center of the system, and that the beams are accelerated very close to this object.

The conclusion is that phenomena as rich and complex as those discovered astrophysically can only be understood if we have the opportunity to observe the same objects several times in different ways, and if we can observe in many different portions of the electromagnetic spectrum. Since the theories that are proposed 
to explain observations in one part of the spectrum often make predictions that are testable only in another portion of the spectrum, much of the scientific momentum generated by discoveries would be lost if these tests could not be performed within a reasonably short time. These conditions mean that we need long-lived observatories.

The long-lived observatory program includes The Hubble Space Telescope (HST), the Gamma Ray Observatory (GRO), the Advanced X-Ray Astrophysics Facility (AXAF), and the Space Infrared Telescope Facility (SIRTF). This combination will make possible high-sensitivity observations over 13 decades in wavelength from $10^{-14} \mathrm{~cm}$ to $0.07 \mathrm{~cm}$. The power of this capability is illustrated in Figure 1. Quasars more than $10^{4}$ times fainter than 3C 273 will be accessible to all the observatories. The characteristics of each facility and the profound impact each will likely have on pursuing the goals of astronomy and astrophysics are briefly described below.

\section{The Hubble Space Telescope}

The Hubble Space Telescope, initially operating over the decade of wavelength from 0.12 to $1.1 \mu \mathrm{m}$, will be the first Great Observatory. Its launch with the Space Shuttle will be the culmination of two decades of planning and development. The $11,250-\mathrm{kg}$ satellite has a length of $\mathbf{1 3 . 1} \mathrm{m}$ and diameter of $4.28 \mathrm{~m}$ (see Figure 2 ). The optical quality of the primary and secondary mirrors is believed to be the finest ever achieved for large optics. The optics should be capable of obtaining an angular resolution of better than 0.1 arcsec at visible and ultraviolet wavelengths. The combination of large collecting area and high angular resolution (and associated rejection of sky background) will allow the HST to image point sources about 40 times fainter than is possible from the ground. The image quality of the HST, which is 10 to 20 times better than is generally possible from good ground-based sites, will allow the resolution and isolation of fine details in extended sources such as galaxies, nebulae, star clusters, and planetary disks.

The initial complement of HST instruments provides imaging, spectroscopic, and photometric capabilities. Included are two versatile imaging cameras (the Wide Field Camera and the Faint Object Camera), two spectrographs (the Faint Object Spectrograph 


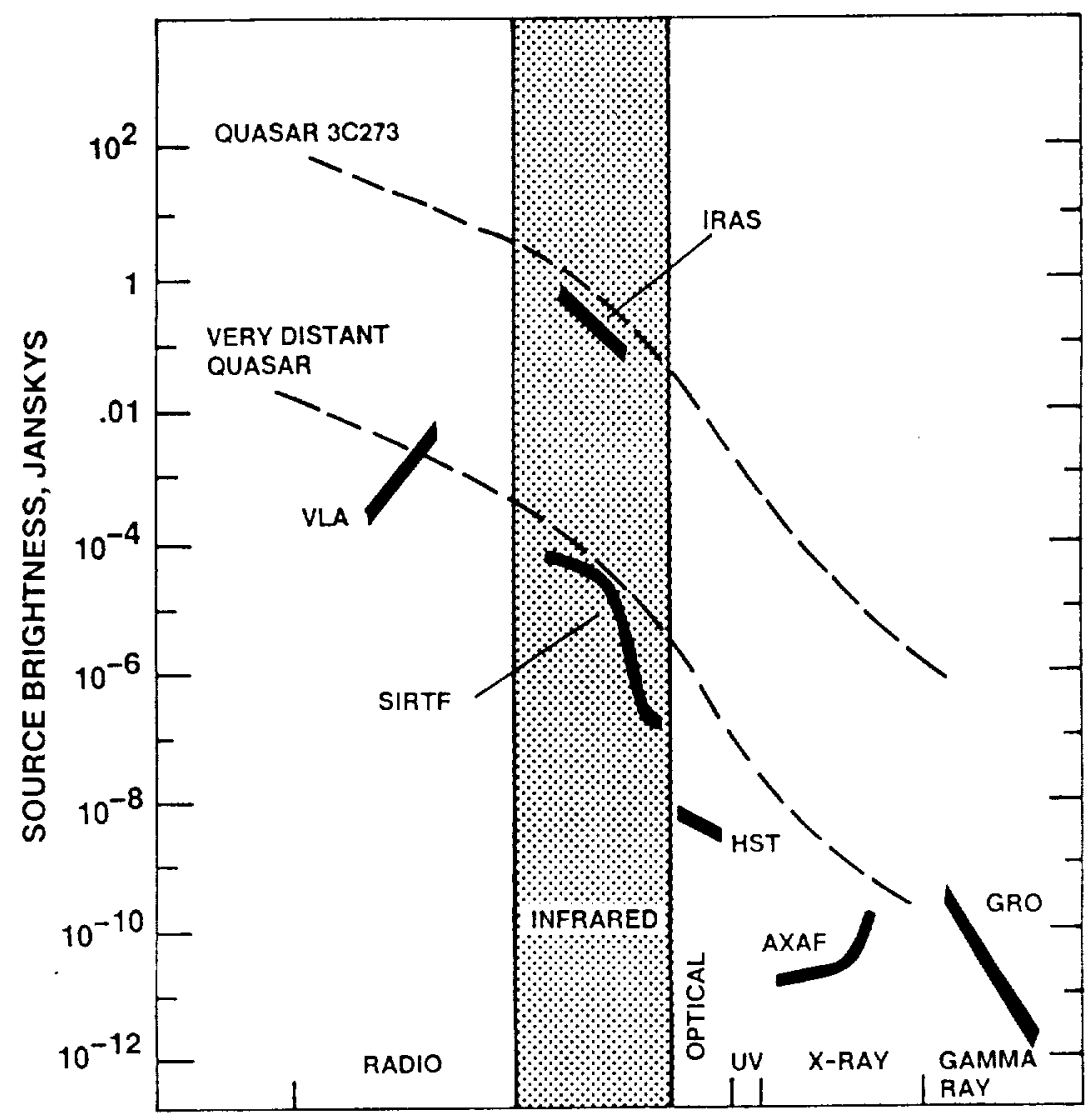

ELECTROMAGNETIC SPECTRUM

FIGURE 1 A plot of the observed brightness of $3 C^{273}$ and the predicted brightness of a similar but much fainter quasar compared to the sensitivities of different instruments at different wavelengths.

and the High Resolution Spectrograph), and the High Speed Photometer. In addition, the fine guidance system will be capable of supporting high-precision astrometric observations.

NASA will oversee the complex operations of the HST through the operations and control centers at the Goddard Space Flight Center and the Space Telescope Science Institute (STScI). Goddard will control spacecraft housekeeping and control functions and the Institute will be in charge of scientific operations, including the allocation of observing time to the international scientific community. 


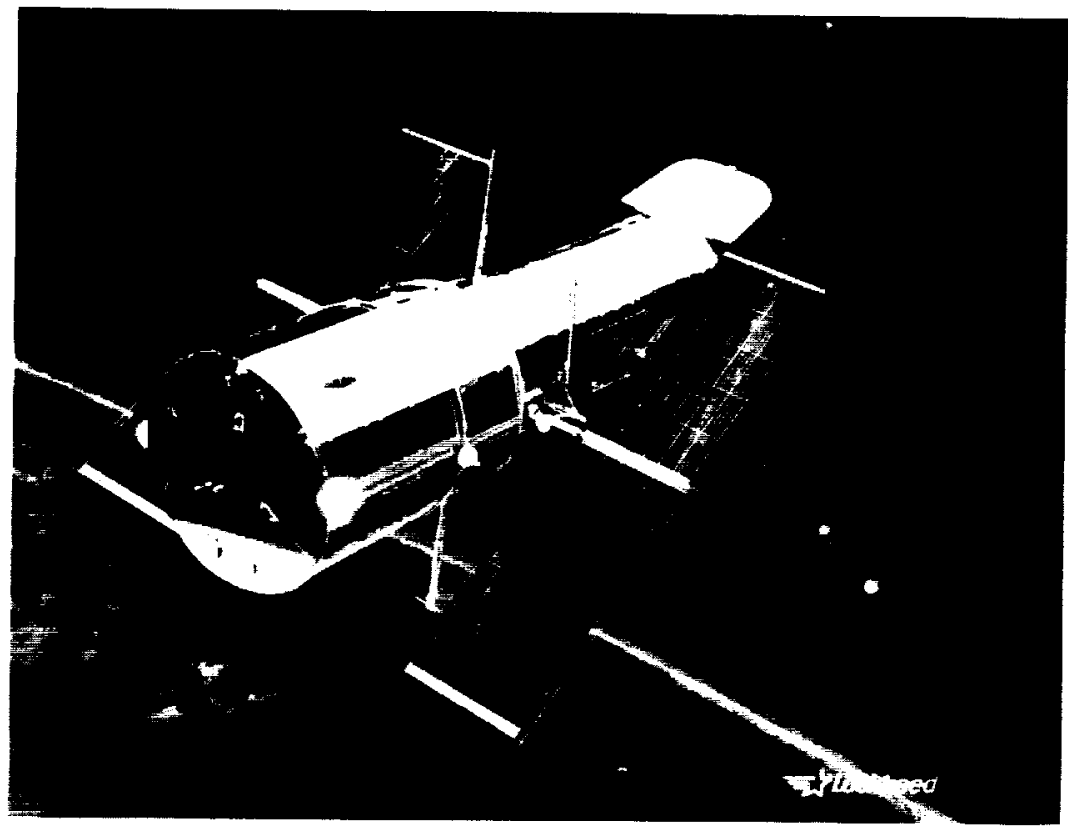

FIGURE 2 A photograph of the Space Telescope.

Observing time requests for the HST are expected to be oversubscribed by about a factor of 10 . The HST will be used to study an extremely wide range of astronomical problems ranging from direct high-resolution imaging of planets in the solar system to measures of the distance scale of the universe. In 1984, six working groups representing the various subdisciplines of the international astronomical community were asked to identify the most important large projects that should be carried out early in the HST mission. Briefly listing and overviewing the results for this study helps to illustrate the profound impact the HST will have on twentieth-century science. The recommended key projects are as follows:

Distances to Galaxies and $H_{0}$. The extragalactic distance scale and an accurate value of the Hubble constant, $\mathrm{H}_{0}$, are fundamental for almost every problem is cosmology. The Hubble constant measures both the size and the age of the universe. In this project the HST will be used to calibrate the distance scale and to evaluate $\mathrm{H}_{0}$ to an accuracy of better than 10 percent.

$$
\begin{gathered}
\text { ORIGINAL PAGE } \\
\text { BLACK AND WHITE PHOTOGRAPH }
\end{gathered}
$$


Medium Deep Survey. The ability of HST to reach very faint objects with relatively short exposures makes possible a survey of a significant area of the sky that should provide important information concerning the content of the solar system, the galaxy, and the universe.

Quasar Absorption Lines. A survey of absorption toward distant quasars in the ultraviolet part of the spectrum should provide a wealth of information about the intergalactic medium, galaxy halos, and quasars. A comparison of absorption seen toward nearby objects with absorption toward distant objects would yield clues about the evolution of the intergalactic medium as the universe expands.

Primitive Bodies in the Solar System. Asteroids and cometary nuclei will be observed in order to study asteroid morphology and their geophysical properties and to explore the chemical diversity of cometary nuclei.

Environments of Nearby Stars. Imaging and astrometric observations of nearby stars will be made in order to search for faint stellar or planetary companions and protoplanetary disks.

Ages of Globular Clusters. The uncertainty in the ages of globular clusters should be reduced to 5 to 10 percent by reducing the uncertainty in the distances to the clusters. This result will have very important cosmological implications since globular clusters appear to be nearly as old as the universe itself.

Evolution of Stellar Populations. Representative stellar spectra covering a wide range of age and metallicity are to be obtained to permit investigations of galaxy evolution and formation.

Intensive Supernova Study. Several bright supernovae will be followed from discovery through their decline to invisibility in order to better understand these catastrophic explosions and the return of processed matter to the interstellar medium.

Morphology of Distant Galaxies. The HST cameras will be used to image a sample of clusters of galaxies over the redshift inverval $0.3<z<1.0$ in order to investigate the evolution of stellar populations, star-formation rates, galaxy morphology, and cluster structure with age.

Imaging and Spectroscopy of Nuclear Regions of Active Galaxies. The nearer active galaxies will be studied in order to determine the properties of the nuclear gas, nuclear stellar component, synchrotron jets, and the central energy source. 
Immediate Environments of Quasars. HST will be used to obtain high-resolution images of a sample of QSOs in order to determine the types and evolutionary states of the associated galaxies.

Ultra-Deep Survey. Two to four fields at high galactic latitudes will be imaged intensively in order to provide information on star counts, supernova rates, and galaxy morphology at ultra-faint levels.

\section{The Gamma Ray Observatory (GRO)}

The Gamma Ray Observatory will be a Shuttle-launched freeflyer with a scientific payload weight of about $6000 \mathrm{~kg}$ (see Figure 3 ). It has a design lifetime of about 2 years. However, the facility may operate for a considerably longer period of time and will form part of the complement of long-lived observatories if it is maintained by the Space Shuttle or Space Station.

The GRO contains four experiments whose common goal is the detailed study of the celestial gamma-ray spectrum from 0.05 to $3 \times 10^{4} \mathrm{MeV}$, almost six decades in energy. Each instrument has been chosen such that its sensitive regions overlap those of its nearest neighbors, thus allowing complete continuity of spectral measurements. The photon sensitivity over the entire six-decade range will be improved by more than an order of magnitude over the best previously flown instruments. Significant improvements in angular resolution will also be made.

The GRO was originally implemented as a PI-class mission with a limited lifetime, and the development has continued with a 2- to 3-year mission as the baseline. However, because the scientific rewards may be very great, NASA has taken action to ensure that GRO can be maintained and repaired on orbit by the Space Shuttle or the Space Station, and has included on the spacecraft the capability for replenishment of the on-board propellent used for orbit maintenance. Only one of the instruments on GRO uses any consumables, and this instrument also has an expected lifetime well beyond the baseline 2- to 3-year mission. Therefore, it is reasonable to expect that GRO will remain extremely productive scientifically well beyond the baseline mission. Because of the great potential scientific return, we recommend that NASA establish plans for extending the lifetime of the GRO mission and to have a vigorous guest investigator program. Gamma-ray astronomy occupies a unique position in the search for understanding 


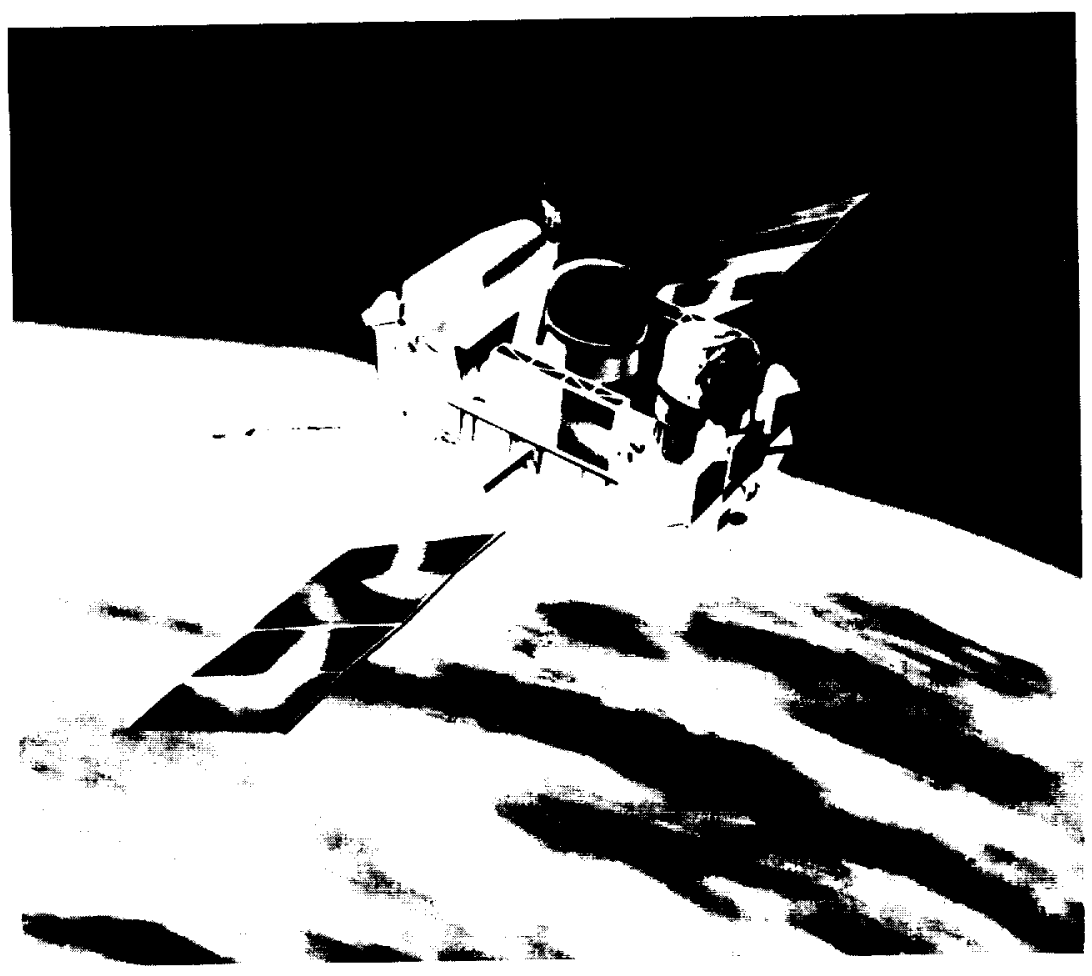

FIGURE 3 A photograph of the Gamma Ray Observatory.

the universe. Gamma rays are produced in a wide variety of astrophysical processes that would otherwise remain unobservable. These processes include nuclear reactions, matter-antimatter annihilation, elementary particle decays, and some general relativistic effects. The great penetrating power of gamma rays allows them to reach the top of the earth's atmosphere from almost anywhere in the universe. Among the problems to be addressed by the GRO are the formation of the elements in the universe, the structure and dynamics of our galaxy, the nature of pulsars, the possible existence of large amounts of antimatter in the universe, phenomena occurring in the nuclei of galaxies (especially quasars), and the origin and evolution of the universe itself.

The discoveries in gamma-ray astronomy have paralleled those in other new branches of astronomy in that the unexpected results

\author{
ORIGINAL PAGE \\ BLACK AND WHITE PHOTOGRAPH
}


have been as significant as those that had been predicted in providing new insights into a number of astrophysical problems. For example, it has been found that some pulsars emit several orders of magnitude more energy in the form of gamma-rays than in the form of radio waves. The quasar $3 \mathrm{C} 273$ appears to radiate as much energy in gamma-rays as in any other form of electromagnetic radiaton. Many energetic gamma-ray sources have been found that at present have not been correlated with objects observed at other wavelengths. Furthermore, intense bursts of low-energy gamma rays have been detected, the origin of which remains a mystery. In all these cases, the objects are not likely to be fully understood without a thorough knowledge of their gamma-ray emission because this emission often represents a significant fraction of the total radiated energy. The understanding of gamma-ray-luminous sources is one of the most important open problems for all astronomy. lowing:

The specific scientific objectives of the GRO include the fol-

1. A study of discrete objects such as black holes, neutron stars, and objects emitting only at gamma-ray energies.

2. A search for evidence of nucleosynthesis, the fundamental process in nature for building up the heavy elements.

3. The exploration of our galaxy in gamma rays in order to study the origin and dynamic pressure effects of the cosmic-ray gas and the structural features revealed through the interactions of the cosmic rays with the interstellar medium.

4. A study of the nature of other galaxies as seen at gammaray wavelengths, with special emphasis on radio galaxies, Seyfert galaxies, and QSOs.

5. A search for cosmological effects, through observations of the diffuse gammo-ray radiation, and for possible primordial black hole emission.

6. Observations of gamma-ray bursts, their luminosity distribution, their spectral and temporal characteristics, and their spatial distribution.

\section{The Advanced X-Ray Astrophysics Facility (AXAF)}

The Advanced X-Ray Astrophysics Facility will be a 1.2-m aperture grazing incidence $X$-ray telescope (see Figure 4) designed 


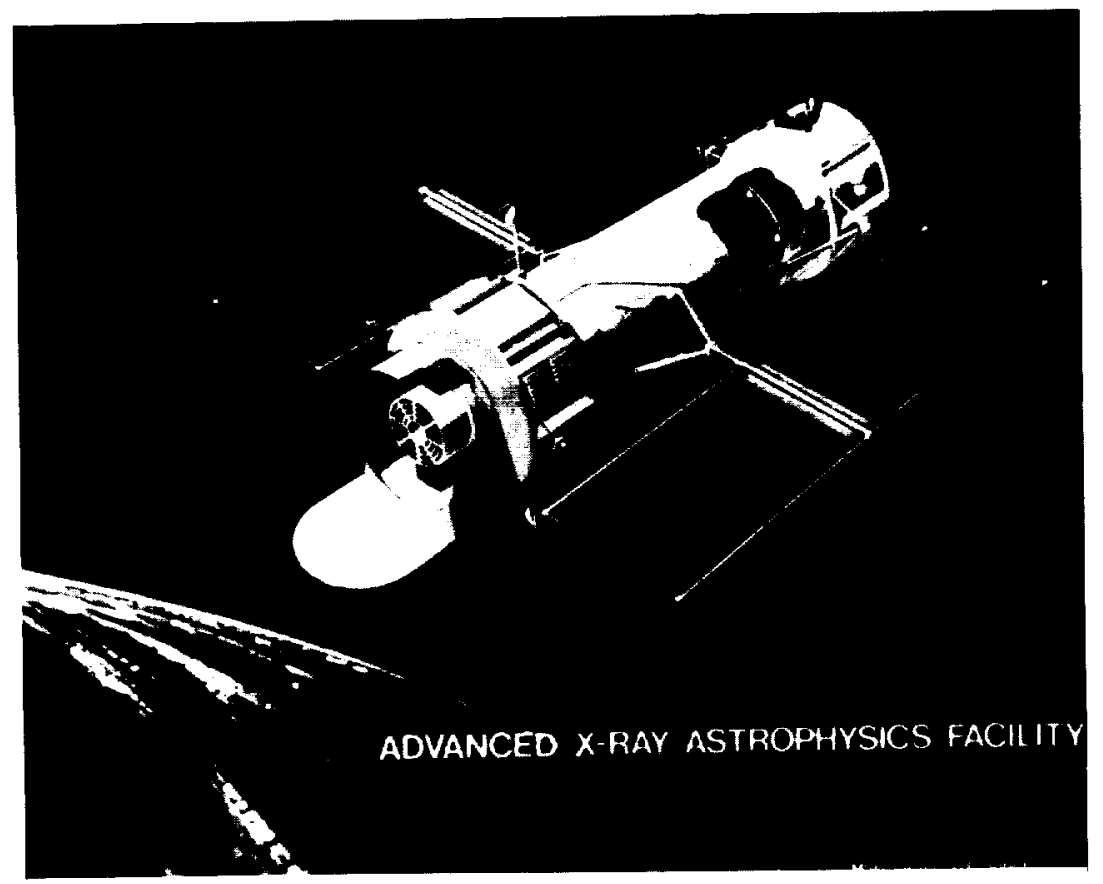

FIGURE 4 Artist's conception of AXAF in space.

to address fundamental questions in astronomy and physics. The importance of AXAF derives from its high sensitivity, thereby exploiting the fact that many of the fundamental processes in space are associated with high-energy particles that radiate mainly $\mathrm{X}$-rays.

AXAF is the successor to the Einstein X-ray Observatory that was flown from 1978 to 1981 and made significant discoveries in astronomy. Like Einstein, AXAF will have special mirrors capable of forming images of astronomical objects in X-ray light. AXAF, however, will go far beyond Einstein in capability. AXAF will have $\sim 10$ times the angular resolution, $\sim 100$ times the sensitivity for imaging, and $\sim 1000$ times the sensitivity for high-resolution spectroscopy. If the history of science is any guide, such a remarkable increase in sensitivity over the previous leading instrument virtually guarantees that AXAF will make major advances in our understanding of nature.

\author{
ORIGINAL PAGE \\ BLACK AND WHITE P'InTกCRAPH
}


Among the astronomical objects AXAF will observe are neutron stars, black hole candidates, the debris remaining from the explosions of massive stars, quasars, the centers of active galaxies, and hot gas in individual galaxies and in clusters of galaxies. Some of the physical processes revealed by the X-ray emission from these objects include the behavior of matter under extreme physical conditions (such as the $10^{12}$ Gauss magnetic fields on neutron stars), the energy transport mechanisms in stellar interiors, the acceleration of particles by shock waves, the nuclear reactions in stars, the evolution and explosions of massive stars, the rate of expansion of the universe, the distribution of the puzzling invisible matter (called dark matter) that seems to pervade much of the universe, and the formation and evolution of structure in the early universe.

In recent years, the partnership between astronomy and physics has grown closer than ever before. The ultimate fate of the universe could be discovered by the careful measurement of distances to galaxies. The existence of exotic particles predicted by new supersymmetric theories in particle physics might be confirmed or ruled out by their gravitational effects on hot gas in galaxies. The equation of state of matter at densities far greater than any on earth can be tested by monitoring the radiation from neutron stars. These investigations concern some of the most fundamental questions in science today.

AXAF is a mission of reconnaissance and observation. Vast numbers of $\mathrm{X}$-ray-emitting astronomical objects will be studied and characterized. AXAF data will stimulate work in theory, modeling, and simulation. Detailed studies of astrophysical plasmas found in stellar coronae, supernova remnants, and clusters of galaxies are expected to provide new insights into the behavior of matter under a variety of densities, temperatures, and magnetic and gravitational fields not achievable on earth. Indeed, the plans for AXAF have already stimulated theorists both in this country and abroad to investigate problems that can be critically tested with AXAF. For example, the study of how hot gas in galaxies cools-which is intimately related to the formation of stars-has been motivated by the potential observations of AXAF. Finally, AXAF is truly a mission of exploration and discovery, with its power to reach the early universe (looking out to greater distances corresponds to looking back in time), with its ability to extend high-resolution spectroscopy by a large factor, and with its novel 
ability to obtain high spatial and spectral resolution simultaneously.

\section{The Space Infrared Telescope Facility (SIRTF)}

The Astronomy Survey Committee in 1982 strongly urged that NASA proceed with what was then called the Shuttle Infrared Telescope Facility, or SIRTF, a 1-m class cryogenically cooled instrument designed to make observations in the range from 2 to $3004 \mathrm{~mm}$ while mounted in the Shuttle bay. At that time SIRTF was part of the Spacelab program, to which NASA had an ongoing commitment. NASA restudied SIRTF, and decided to redesign it as a free flyer, called the Space Infrared Telescope Facility. In this mode, SIRTF will be able to study the emission from stars and planets, newly forming galaxies, and emission by quasars in a wide spectral band that is currently inaccessible. Instruments have now been chosen for SIRTF that cover over two decades of wavelength from 2 to $700 \mu \mathrm{m}$. These instruments are redesigned and reconfigured as progress is made in infrared detector technology.

SIRTF (see Figure 5) is currently planned to have a $0.85-\mathrm{m}$ aperture, with optics and detectors cooled to less than $7 \mathrm{~K}$ by superfluid helium. SIRTF could be carried into low orbit by the Space Shuttle and an additional propulsion system would boost it to an orbital altitude of between 700 and $900 \mathrm{~km}$. IRAS showed that the environment at this altitude is benign for a cooled telescope; therefore, SIRTF's performance will not be degraded by thermal radiation from the earth or the sun, by cosmic rays or trapped energetic charged particles, or by contamination from the residual atmosphere. The SIRTF telescope is compatible either with a free-flying spacecraft or with operation from a standarized platform that might be part of the Space Station program.

SIRTF will be at least 1000 times more sensitive than the Infrared Astronomical Satellite (IRAS). SIRTF will be a true observatory with a versatile group of instruments including (1) a wide-field, high-resolution camera covering the 3 - to $30-\mu \mathrm{m}$ region with large arrays of detectors; (2) an imaging photometer, with small arrays of high-sensitivity detectors covering the wavelength range 3 to $700 \mu \mathrm{m}$; and (3) a spectrograph operating from 2.5 to $200 \mu \mathrm{m}$ with spectral resolutions of 2 and 0.1 percent. The instruments will be built by teams selected in July 1984 . 


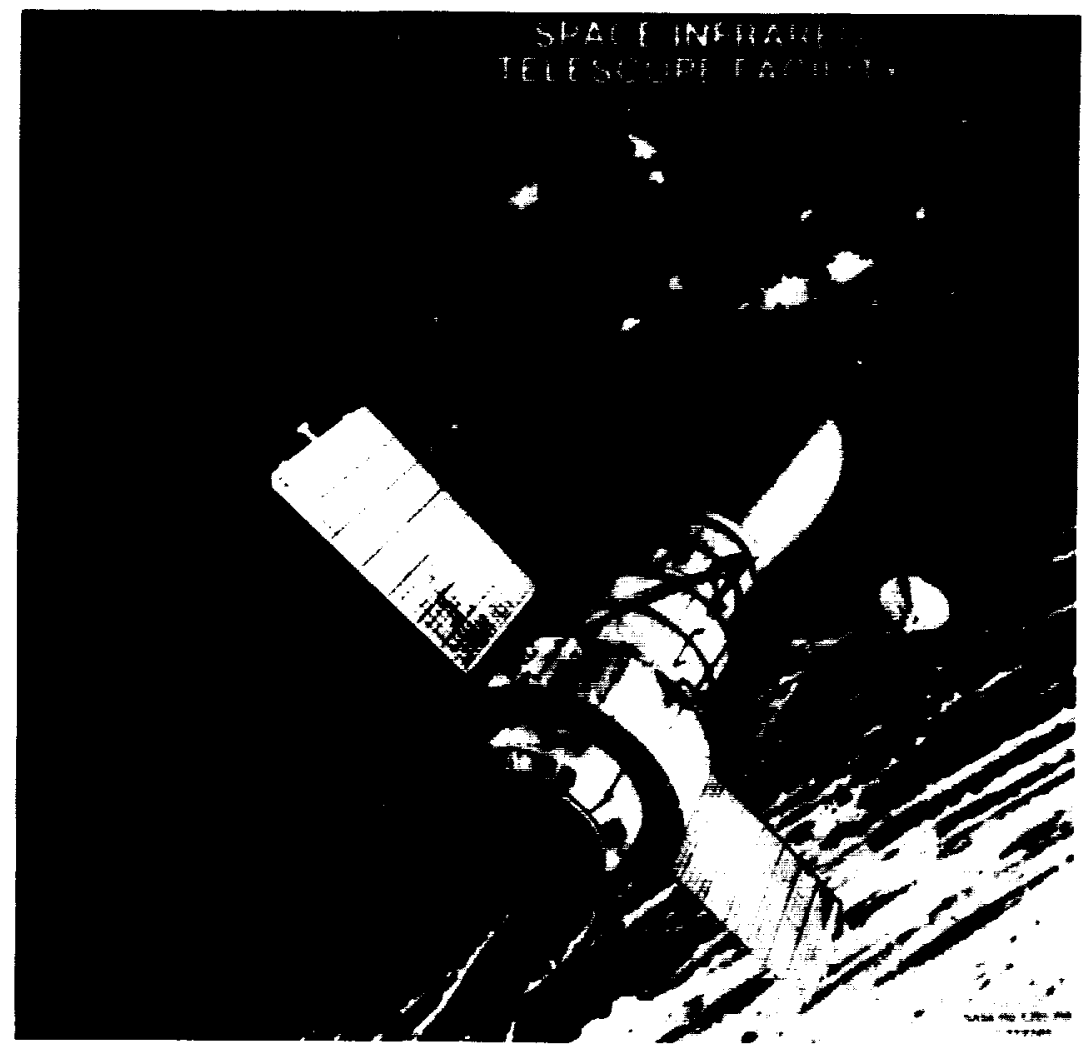

FIGURE 5 Artist's conception of SIRTF in space.

Infrared astronomy reveals cool states of matter-solids and molecules. Various mechanisms heat cosmic solids to temperatures between $3 \mathrm{~K}$ (determined by equilibrium with the cosmic background radiation) and $1500 \mathrm{~K}$ (above which all but a few of the most refractory solids evaporate). Over this temperature range, most of the radiated energy lies in the infrared. The bulk of strong molecular spectral lines also lie in the infrared. Cosmic solids and molecules are of special importance in the study of such low-temperature environments as regions of star formation, planetary surfaces and atmospheres, and sites where the first steps toward formation of life may be occurring.

The infrared radiation recorded by SIRTF can penetrate the dark clouds of matter in space where stars are forming. Since,

\author{
ORIGINAL PAGE \\ BLACK AND WHITE PHOTOGRAPH
}


moreover, newly forming stars emit most of their energy in the infrared, SIRTF will be a superb tool for finding such stars. With its spectroscopic capability, it will also be possible to study the emissions by specific molecules, such as carbon monoxide, that are abundant in such objects. These emissions will yield detailed information about the density and temperature near the newly forming stars, enabling us to reconstruct the process by which they are forming.

The last discovery of a planet was that of Pluto in 1930. The discovery of a planet orbiting another star has eluded us until now, in spite of the great advances in instrumentation that have occurred in the meantime. The reason is not hard to find: if Pluto is the last planet in the solar system, the next one must be sought in the vicinity of a nearby star, and the nearest stars are 6000 times more distant than Pluto. The potential of SIRTF carrying out a search for planets beyond the solar system has been carefully studied, because planets are much brighter in the infrared relative to their parent stars than they are in the visible wavelengths. Recent studies of the application of a technique known as "super resolution," a method to measure small angular sizes, indicate that SIRTF should be able to separately image planets like those in the solar system if they are in orbit around nearby stars. In the light of the discovery by IRAS of disks of small particles orbiting stars, it is quite likely that SIRTF will be able to discover planets outside the solar system.

Frequently, the visible radiation absorbed by dust and reradiated in the infrared accounts for virtually the entire luminosity of dust-embedded stars, active galactic nuclei, and even whole galaxies. Thus, SIRTF will provide fundamental insights about cosmic energy sources. Newly born galaxies should be 100 times as luminous as our own, and because of the cosmological redshift effect, most of their radiation should arrive at the earth in the infrared band. Therefore, SIRTF provides a way to find such objects. Calculations indicate that SIRTF should be able to detect newly forming giant elliptical galaxies even if they are so distant that the light left them when the universe was only 4 percent as old as it is now-and this is right in the range predicted for the formation of galaxies. Thus, SIRTF is uniquely qualified to study two fundamental problems in astronomy-galaxy formation and star formation. 
This science program leads to the first three recommendations of our report:

1. We recommend that $H S T$ and GRO be given high priority for launch with the Space Shuttle.

2. We recommend that $N A S A$ establish plans, including a guest investigator program, to extend the mission lifetime of the GRO beyond the currently designed 2 to 3 years.

3. We concur with the recommendations of the Astronomy Survey Committee Report (1980), which urges the construction of $A X A F$ and SIRTF. 


\section{3}

\section{Previous Studies}

There is a variety of published material that bears on the subject of this report. Two reports are particularly relevant both because they are timely and because they reference much of the previously published study work.

\section{THE HORNIG AND GORDON REPORTS}

In 1976, at NASA's request, a committee sponsored by the Space Science Board and chaired by D. Hornig issued a study Institutional Arrangements for the Space Telescope (NAS, 1976) that recommended that an Institute be established for the Space Telescope. The Institute was to "provide the long-term guidance and support for the scientific effort, to provide a mechanism for engaging the participation of astronomers throughout the world, and to provide the means for the dissemination and utilization of the data derived from the Space Telescope."

In February 1981, NASA contracted with the Association of Universities for Research in Astronomy (AURA) to form the Space Telescope Science Institute. The Institute is located on the campus of the Johns Hopkins University, where buildings have been erected and initial staffing has been completed.

In May 1984, NASA asked the Space Science Board to review 
the status of the Space Telescope Science Institute. This review (chaired by W. Gordon) Institutional Arrangements for the Space Telescope-A Mid-Term Review (National Academy Press, 1985), considered whether the original goals and objectives of the Hornig committee needed to be modified. The Gordon report concluded that the original aims remain valid. To quote the report's summary: "[the committee] has formed a favorable impression of the Space Telescope Science Institute carrying out of the functions identified by the Hornig report as well as its making valuable contributions in identifying and undertaking tasks that were not foreseen at the time of the Hornig report. In its short time of existence the Space Telescope Science Institute has created a scientific staff of high stature."

Our report notes, however, that there are differences between the Space Telescope and AXAF and SIRTF. Many of the observations performed by the Space Telescope will be at optical wavelengths where there is already a vast wealth of data accumulated at ground-based observatories. The community that will be served has a centuries-long history with a set of expectations and style very different from those of NASA. In contrast, AXAF and SIRTF are the fruition of recent space projects such as Einstein and IRAS. Also, AXAF and SIRTF have different operational requirements from the Space Telescope. There is no reason for the management schemes of these two facilities to parallel that of the Space Telescope.

\section{THE MARTIN REPORT}

Independent of this study, NASA established a special committee chaired by F. Martin to investigate "Astrophysical Data Operations." The final version of this work is dated April 15, 1986.

The goal of the Martin report was somewhat similar to that of this one-to develop strategies for the optimal management of AXAF and SIRTF. The Martin committee made four major recommendations regarding AXAF and SIRTF; our committee endorses all four of these proposals listed below:

1. Early in the history of a program, there should be established an institutional setting for operations with clearly established lines of scientific and management responsibility. The 
choice of the institutional setting for AXAF and SIRTF should be made in the near future so that technical decisions do not get "locked-in" without appropriate evaluation by personnel with an overall scientific perspective on the project.

2. Simplicity of management should receive very high priority. Consistent with this goal, there should be adequate staffing of the science and operations teams early in the development of a project.

3. NASA should implement a mechanism for regular and intimate interactions among teams from the major space observatory programs.

4. The acquisition, analysis, distribution, and archiving of data should be a central part of mission planning from the beginning.

Both the Hornig and Martin reports reflect the basic philosophy that scientific excellence in the utilization of space observatories will most likely result from peer-reviewed competition for resources, such as observing time, by the widest possible community of astronomers. Both reports have a strong sense that the facilities will be complex to operate and that a dedicated staff will be required to help visiting scientists obtain and analyze data. Finally, both reports stress that an essential ingredient for the success of space observatories is that they must be operated with the confidence and respect of the astronomical community.

Many of our recommendations reflect the consensus opinions in both reports. Therefore, as in the previous studies, we strongly support peer review in the process of reaching decisions on the operations of an observatory.

The most striking difference between the Hornig report regarding the Space Telescope, and the Martin report regarding AXAF and SIRTF is that for the latter two facilities, there is no recommendation for the establishment of a separate and distinct Institute for scientific operations. The Martin committee implicitly felt that AXAF and SIRTF could be optimally operated within current organizational structures. Although the opinion was not unanimous, this committee also agrees that the differences from the Space Telescope are sufficiently great that there is no need to establish new institutes for AXAF and SIRTF in order to ensure their best scientific utilization. 


\section{4 \\ Requirements for a Long-Lived Observatory Program}

Current NASA plans are that the HST and GRO will be launched in 1989 and 1990, respectively. It is hoped that AXAF will be operational by 1995 and SIRTF by 1998. In this context, we make our suggestions for operations and management of these facilities.

\section{SPACECRAFT CONTROL}

Long-lived space observatories place unique system-level requirements on the development and operational aspects of these missions. The optimum strategy for spacecraft control can differ significantly depending on requirements for experiment viewing, communications, support, or unique orbit parameters. It is important to undertake early system studies that identify the cost trade-offs between competing options. For example, requirements for maintenance and repair are strong drivers toward low-altitude missions.

Three-axis-stabilized spacecraft are typically more expensive to design and develop than spin-stabilized spacecraft; however, three-axis capability is essential to the scientific aims for the missions cited above, and this outweighs the additional development costs. For other future missions, different considerations may be 
paramount. For example, the operational cost of a vehicle in geosynchronous orbit can be greatly reduced because of simplified communications interfaces: TDRSS interfaces, on-board recording requirements, and some NASCOM interfaces can be eliminated, as can the ground and spacecraft subsystems needed to support these capabilities. Similarly, the cost effectiveness of repair and servicing in space must be evaluated on a mission-by-mission basis, and with realistic expectations regarding the true cost of manned interfaces. The scientific goal of long-lived facilities may be economically achievable by periodically launching observatories of moderate lifetimes that are not routinely serviced.

Operations and operational costs have a major impact on overall mission activities. The large costs associated with changes at the system level demand that all operational aspects be investigated early. Therefore, we recommend that mission operations and spacecraft control planning be an integral part of the mission activities from the beginning of the program, and that the scientific requirements that affect mission operations be specified early in the program. This requires a dedicated project scientist and science staff with adequate funding to provide full scientific input to the system level design activities from the beginning of the mission.

We further recommend that:

1. Systems studies be undertaken to develop an appropriate mission management structure, and to minimize the number of organizational and subsystem interfaces, consistent with mission objectives.

2. There should be an early definition of a mission systems approach that minimizes overall costs, including the operational phase costs.

3. The history of experiments connected to manned programs reflects considerable cost and time penalties in direct contrast to previous NASA projections. Options for undertaking AXAF and SIRTF with minimal requirements for manned space activities, or even no manned intervention, should be thoroughly investigated. Well-considered utilization of the Space Station for maintenance and repair should be evaluated early in the program.

In this regard, all together, these arguments lead to number 4 of our summary recommendations: 
4. We recommend that $N A S A$ vigorously plan to minimize the operational and refurbishment costs of the entire suite of long-lived space observatories.

\section{MISSION PLANNING}

NASA's major observatory missions will be operated primarily to serve the needs of guest investigators, rather than (PI) science teams who were closely associated with the development of the observatory. Mission operations must therefore be planned and conducted in a manner consistent with the needs of the guest observers. Specifically, the system should be configured to accommodate a broad flexibility in observing approaches. Likewise, the mission planning support systems should be tailored to the requirements of guest observers recognizing their diverse observing needs and their lack of familiarity with detailed constraints imposed by the instrument, spacecraft systems, and orbit configuration. Efficient operation in this context requires that the support systems be significantly automated and the user shielded from unnecessary complexities in the intermediate stages of data processing. The Science Operations Center (SOC) staff should ensure that interfaces with other mission systems (that is, those that interleave different observing programs, plan data capture sequences, and so on) be standardized and rigorously controlled.

The current baselined use of low earth orbit for the longlived observatories in space leads to complex constraints in observing programs that require a significant mission planning activity throughout the lifetime of the observatories. The scheduling of command and telemetry communications contacts, operational inefficiencies, and increased workload on the SOC staff are significantly reduced if preplanned observing programs are utilized, to the exclusion of real-time interactive observing. Typically, the observing sequences should be worked out months in advance, with final command loads generated a week before being uplinked to the spacecraft for execution. Mission operations must also provide a quick response to targets of opportunity, for example, supernovae or newly discovered comets. This could be carried out by suspending planned observing sequences and inserting special programs held in reserve for the advent of the particular class of object. Such modifications need to be accomplished within one 
or two days to be scientifically productive. Targets of opportunity should be limited and infrequent. They impose surges in the planning workload for the preparation of new observations plus they require a plan to resume the suspended program. Finally, missed observations need to be rescheduled at a later time or dropped from the program. The requirement to be able to modify a preplanned program within days has an impact on the sizing of mission planning staff and support hardware regardless of how often it is actually utilized. However, the scientific return from observations of unexpected or extraordinary events is generally considered to justify such a capability. Any further requirement for real-time operational modes that impose even greater costs should be avoided.

The concept of multiple long-lived observatories in space opens the way to observing time-variable objects simultaneously with two or more space facilities as well as observations from the ground. Software in the mission planning network should have features that account for the multiple constraints of other observatories and present to the staff the joint observing options that are available. Such programs might include algorithms to optimize the efficiency of the sequence. Without computerized support in this area, synoptic observing would probably require extraordinary human effort or result in intolerable inefficiencies.

In addition to the mission planning functions associated with simultaneous observations, the overall management of the Great Observatories must provide mechanisms for joint observing proposals and guidelines for the fraction of time available for this mode.

We recommend that:

1. Real-time operations not be a requirement for the major observatories of the future such as SIRTF and AXAF.

2. Single experiment operation be the operating mode so that development and operational costs are minimized.

3. The science requirements relating to mission operations be specified early in the program and that these be controlled in the same way that other mission requirments are controlled.

4. The number of operating modes on each instrument be minimized to those that are clearly scientifically justified. 
5. The requirements/capabilities associated with simultaneous observations be studied early in the mission so that derived requirements (timing, position reference, and so on), can be defined and the techniques and software available from other missions can be identified and utilized where applicable.

6. Suitable mechanisms and guidelines be established for joint observing proposals to avoid situations where only parts of programs are approved or where schedules are not coordinated.

\section{USER SCIENTIFIC SUPPORT}

User support deals with the expertise necessary for assuring the production of useful data products and tools. This section describes the requirements for assuring their availability to users. Spacecraft systems and performance needs to be characterized, particularly with respect to scientific performance of the observatory. The individual instruments need to be understood in detail, their performance characteristics well documented, the calibrations maintained, and the standard data reduction procedures verified. Overall operations of the observatory need to be monitored and evaluated with respect to the scientific quality of the data.

These functions must continue over the lifetime of the observatory, taking into account changes in instrument performance and/or calibration. In addition to these functions, user support must provide observers with various degrees of assistance in understanding their data. Documentation of the observatory systems and assistance in carrying out complex data analysis are examples of this function.

Several options exist for implementation of instrument hardware and data analysis systems that should be considered suitable for a given mission. With regard to hardware development, the traditional approach has been the PI mode. With complex facilityclass instruments there is a near-continuum of options for bringing about all the functions that were traditionally the responsibility of the PI. Included in these options is a central planning organization that established standards and procedures for the observatory and may also serve as the central repository for the software systems and system documentation. Other functions associated with user support are prelaunch activities such as the development of hardware to postlaunch activities such as maintenance of calibration 
data bases. NASA should investigate the advantages of various approaches and make decisions regarding the optimal stategy for each mission accordingly.

As stated in other contexts, it is also important here to identify requirements early in the program. In the user support context, this relates to development of software and the associated computer systems on which the software is used. It is important to ensure that software system planning will result in software that can support the instruments during the several phases of the program, including instrument testing, spacecraft integration testing, mission operations, and data analysis.

For the large missions that primarily support guest investigator operations, it is clearly important to have dedicated personnel in the postlaunch phase who are intimately familiar with all aspects of each instrument and who can provide expert guidance to prospective guest investigators. The location of such experts can vary. They could be associated with instrument development teams, they could be an integral part of a centralized scientific operations facility (similar to the Space Telescope Science Institute), or they could be part of a NASA center. NASA should carefully consider the optimum approach based on the types of observational support provided, the user community, the role of instrument development teams in the postlaunch era, and the state-of-the-art systems that are or will be available to provide communications between the several groups involved in the operations and the data handling and analysis. The important point that must be continually emphasized is that the establishment of a core group of experts must take place early in a program lifetime. The role of such a group begins at the start of the development phase of an observatory and not after it it launched and operational.

These arguments lead to recommendations 5 and 6 :

5. We recommend the active involvement of the broad community of users in all phases of the long-lived observatory program.

6. We recommend that each long-lived observatory have a dedicated science operations staff with a well-defined scope of responsibilities in order that each observatory can be utilized to its full potential. 


\section{DATA HANDLING}

Our recommendations are consistent with clear trends emerging from similar studies that have been performed during the last few years for specific or interdisciplinary research programs. In particular, we refer to the two Committee on Data Management and Computation reports $(1982,1985)$, Trends in Planetary Data Analysis (NASA 2333, 1984), and the report of the Martin Committee on Astrophysics Data Operations (NASA TM 87785, 1986).

In all such studies the term "user" is synonymous with researcher, but the term "system" is less clearly defined. Since users may, at different times in a single program or in attempting to utilize data from different programs at the same time, require data or tools from diverse sources, the term "system" will be used in reference to all those functions for which NASA must have the ultimate responsibility, independent of the detailed manner in which those functions are supplied. For specific missions (for example, AXAF and SIRTF), large portions of this responsibility may be delegated to mission-specific Science Operations Centers (SOCs), especially during their planning and active phases.

The first responsibility of the system is to collect and process the data in a form suitable for scientific analysis by the user. Rote activities in this process (for example coping with data dropouts) may be performed outside the purview of the mission-specific SOC, but functions that are potentially more subjective (for example, removal of instrument signatures) will certainly require the involvement of the SOC staff in both the design of the necessary corrections and their implementation. The precise mix of functions performed at the SOC is a matter for study in the case of each particular mission, but we recommend early SOC establishment and the involvement of SOC personnel in such studies.

Further responsibilities of the system to the users include the management of data distribution activity, and ensuring the availability of software tools necessary for both data access and data analysis. The direct involvement of scientists is most important in the latter function. The data analysis software must have at least two levels: one tightly controlled by the system in order to provide a prompt "standard" first-cut analysis product to original users (and later to archival users), and the second accessible to users for in-depth analysis. The SOC staff and hardware PIs must have specific documented tasks for the former level. The analysis 
software system needs to have a more open structure than the standard processing system so that users' experience in dealing with the data can be absorbed and passed on to other researchers. A system with a well-defined core set of functions will be initially required and supplied by the system; later this may well be augmented with software developed and voluntarily furnished by users in accordance with standards set up by the SOC.

The development of software systems for analyzing astronomical data is a complex task that requires joint efforts of hardware and software specialists. Knowledgeable scientists who are involved in the operational aspects of data analysis are needed to develop complete specifications for software systems. Furthermore, as a precaution against errors in the basic analysis software, verifications should be performed by scientists not involved in the original development of the routines.

The key element in the evolving concept of user interactions with space data is that the user can access (almost) everything he or she might need at a home institution. This concept presupposes that while a typical investigator may sometimes prefer to visit a "center of excellence" in order to interact personally with individuals, one also has the option to become an expert user without leaving the office. The necessary computer hardware would normally be supplied by one's home institution (for example, a work station and connections to a larger institutional machine for storage or CPU), but the "system" will furnish access to a fast information network and will supply all the software and protocols. As much as possible, such programs should be in a high-level language compatible with a broad spectrum of commonly used machines and operating systems.

It is expected that the user will be provided with a friendly but intelligent interface, so that while novice users can have all the menu aids and prompts that they may require, expert users will be able to use cryptic commands and interact with the system with greater flexibility, and even some access to lower-level languages. A user should be able to draw upon a variety of documented analysis routines (such as IRAF), as well as data in standard formats (for example, FITS). The utilization of standard languages and protocols must be rigorously enforced, but the system must be flexible enough to accommodate innovative data handling and analysis tools, especially in the active phases of missions when the data have not yet been permanently archived. 
Independent of the exact nature of the individual data base, the user must be guaranteed remote access by the system. NASA must therefore not only supply the network, but also manage the design, operation, and protocols. The data bases must contain scientific data (both raw and processed), ancillary data (engineering, calibration, and so on), and such additional information as might be necessary (special processing requirements that are not easily parameterized). NASA must charge the highest-level data storage elements for each discipline (the Data Centers) with the task of supplying catalogs and directories with multilevel entry, and smart sign-on procedures for proprietary and/or appropriate access to specific data bases or components.

Data from the Great Observatories can easily be accommodated within these general considerations. During their operation phases, the SOCs will serve as Data Repositories, with data access guaranteed by NASA to qualified users. The archive will be distributed to Data Centers (the Space Telescope Science Institute is an obvious candidate) as well as to Active Data Base locations as appropriate, with the condition that users have the option to physically visit these locations if they choose, or to have access to all the information and tools that they require for expert analysis at their disposal via the information network.

\section{FACILITY SUPPORT AND FACILITY LIFETIME}

Prior to Space Telescope, scientific spacecraft were designed to operate for a fixed period (typically two years), but were then operated as long as useful data could be obtained from them. Examples are the Uhuru, HEAO, and IUE missions. Functioning spacecraft in low earth orbit were operated until they reentered the atmosphere, while some spacecraft in higher orbits were operated for more than a decade. The effective cost for extending the lifetimes in this way was quite low.

However, the advent of the Space Shuttle has created the opportunity for planned servicing of scientific spacecraft. As a result, HST, AXAF, and SIRTF are currently designed as longlived observatories and depend upon the availability of the Space Shuttle and an Orbit Maneuvering Vehicle or Orbit Transfer Vehicle for maintenance, emergency repairs, and even replacement of major components. As noted above, GRO has implemented a maintainability program that also provides the opportunity for an 
extended mission, which this report endorses. There are technical and schedule constraints associated with on-orbit servicing that affect the cost advantages of servicing. Recent studies for HST indicate that it is more cost effective to perform only necessary servicing in orbit, rather than the once proposed 5-year regular servicing via retrieval, ground servicing, and relaunch.

Nevertheless, despite this saving, the total operations expenditure for HST will still be about $\$ 160$ million (1986\$) per year. Therefore, extreme care must be exercised in specifying the lifetime of future observatories. We shall address general requirements and cost considerations in this section, and return to recommendations for cost control later.

The fundamental requirement of a lifetime longer than the failure of the first critical component implies the need for servicing. Spacecraft and instrument components cannot be designed to have arbitrarily long guaranteed lifetimes, and, in most cases, nominally longer-lived "high-reliability" components can be very expensive. Cost trade studies to determine the optimum design lifetime of critical components were carried out during the phase-B study for AXAF. It was concluded that 3-year to 5-year servicing intervals will be necessary. In addition to servicing of critical spacecraft systems at few-year intervals, cryogen replacement is necessary for both AXAF and SIRTF, and at least for AXAF, experiment replacement or upgrade is anticipated. Therefore, if these are to be long-term observatories, on-orbit maintenance will be required. A low-cost approach is to schedule routine maintenance well in advance, to perform maintenance only if required, and to accept extended periods of "down-time" if unexpected failures occur.

It has been suggested that moderate-lived missions with no servicing be considered as an alternate approach. In this concept, mission lifetimes of 3 years to 7 years are envisioned, with launches of replacement observatories scheduled on 10-year centers. In order for this to be a viable plan, the cost savings achieved by not planning for service nor carrying such servicing out, must equal or exceed those of servicing. At this time, there is little experience with the true costs of on-orbit servicing. Cost estimates for servicing on AXAF and SIRTF will need to be refined once HST is in orbit and maintained. It is expected that there will be some one-time service costs borne by the HST program that will not recur for AXAF and SIRTF. For example, the carriers used to transport hardware to an observatory can be common. 
Logistical operations may be shared as well and thereby reduce costs. Initial estimates show that moderate-lived mission scenarios are similar in cost to long-lived programs when integrated over long periods of time, or equivalently, the cost per observing unit of total exposure is not greatly different. These estimates are worth refining; however, the most relevant new data can only come after experience operating HST and should not be a basis for delaying the start of the AXAF and SIRTF programs.

A key element in the scientific success of a mission is the existence of a group of individuals who are committed to and responsible for the scientific planning and for the ultimate use and operation of the observatory. These individuals can be centrally located at an "Institute" such as the Space Telescope Science Institute, or more loosely distributed at Science Operations Centers, PI groups, or data repositories. It is essential that continuing involvement of such individuals be maintained in order to assure responsibility. It is also essential that they be delegated sufficient authority to carry out their role effectively and that simple lines of authority be clearly established as early in the mission lifetime as possible. Each mission need not have these individuals organized in the same manner, but each must be organized early enough to accept "Ownership" for the system that they will operate. Rules for oversight, awards, penalties, and eventual dissolution of each individual part of the program should be clearly established, consistent with the necessity to properly archive the data for general access. Since there is a natural instinct to preserve a comfortable status quo, we recommend that regular reviews of all programs be conducted by a single high-level peer-review committee with oversight responsibility for the entire menu of space astrophysics observatories, rather than with scope limited to only one of them. The details associated with the chartering and reporting procedures of this committee are much less important than the rationale for its establishment. Other review processes that impede the work of the group should be kept to an absolute minimum.

In the light of current HST experience that suggests the continuing facility support for a single observatory can be very expensive, we recommend that the scope for this oversight committee include the review of all decisions pertaining to a maintenance strategy, and new instrument development for all the astronomy observatories in the context of the total program for astrophysics. This should include a regular review of the ongoing importance 
of each mission and the establishment of impartial criteria that would allow for the orderly shutdown of observatories or support facilities that have outlived their usefulness, or have become too expensive to repair or to operate in comparison with their value to the overall research program.

\section{SCIENTIFIC MANPOWER ISSUES}

As the several disciplines in space astrophysics have matured, large observatories have become necessary to continue the progress initiated by the previous, smaller missions. In the recent past, the frequency and scope of the smaller missions was adequate to permit many active university, government research laboratories, and NASA Center groups to become intimately involved with experiments from conception, through instrument development, to data analysis. Now, however, new issues concerning manpower training and support for these disciplines arise due to the large costs, extended development times, and reduced mission opportunities associated with the observatory missions under study and/or development. Important questions are:

1. How are stable university/NASA center research groups to be supported effectively in the observatory era?

2. Can suitable mechanisms be developed that involve graduate students in aspects of these missions?

3. Is the current Supporting Research and Technology (SRT) program effective in leading to the development of experiments that are suitable for facility-class missions?

4. How can stable funding be assured for postdoctoral positions?

5. Can Guest Investigator progams be altered to provide different styles of support?

6. How will theory and interpretation of the available data be funded?

As the missions become long-term and broadly based, changes can be introduced. Guest Investigator programs should be restructured to provide stable support for users of long-term observatories. We recommend that a significant fraction of the resources allocated to Guest Observer programs be used to support programs that will continue for at least 3 years.

This approach will provide continuity of funding to research 
teams so they can dedicate the effort that is required to understand the complex data from these facilities, and will allow the teams to plan for graduate student and postdoctoral involvement in an effective manner. Also, theory programs should be explicitly associated with observatory class missions. The interaction between theory and observation will enable astronomers to develop the deepest understanding of the phenomena they are studying.

\section{INTERNATIONAL CONSIDERATIONS}

The American effort in astronomy and astrophysics from space observatories started as the exclusive domain of NASA with missions such as the Orbiting Astronomical Observatories, Uhuru, and Einstein. However, in recent years, the trend has been for a greater international presence in space, and in many areas of space science the foreign ventures are far more vigorous and productive than those of NASA. Until AXAF and SIRTF are built, the Europeans will dominate X-ray and space-based infrared astronomy with their ROSAT and ISO missions. In addition, the Soviets and the Japanese are apparently also pursuing ambitious space astronomy programs. With an increased international presence in space, it is of crucial importance to avoid duplication and to foster cooperation rather than direct competition. The benefits of a cooperative approach go well beyond basic financial considerations. The best science can be obtained by involving the best scientific talent regardless of the nationality of the scientist.

There are numerous examples of highly successful cooperative space astronomy missions. The International Ultraviolet Explorer (IUE) and Infrared Astronomy Satellite (IRAS) illustrate the economic and scientific value of direct cooperation in the construction and operation of an astronomical satellite. Other missions such as the Einstein Observatory had guest observer programs with a vigorous international component. The scientific productivity of all these missions was greatly enhanced because of the participation of a large number of talented scientists and engineers from countries all over the world.

The benefits of international cooperation must be weighed against the disadvantages. International cooperation can seriously complicate the management of a satellite program unless careful measures are taken to simplify the management structure that must span different countries and agencies. Without such 
measures, the increased management and operations cost may actually cancel the cost benefit of having an international partner.

Of the four Great Observatories, the one currently with the most extensive international collaboration is the HST. The European Space Agency (ESA) is supplying the Faint Object Camera (one of six focal plane instruments), the solar panels, and some operations support staff at the Space Telescope Science Institute. In return, scientists from ESA member nations will be allotted at least 15 percent of the HST observing time. This time allocation will be in open competition through a single telescope allocation committee. The results of the common review will be checked to see if the time allocated to the European observers meets the minimum fraction; if not, an adjustment will be made. In the AXAF program, the competitive selection process for the AXAF focal plane instruments resulted in one instrument Principal Investigator team from the Netherlands and one Interdisciplinary Scientist from England, with support for these participants provided by their governments. It is expected that the world-wide astronomical community will become involved with AXAF primarily through the extensive general observer and archival data base programs. The same may be true for the guest observer programs to be associated with SIRTF and possibly GRO. If substantial amounts of observing time are awarded to foreign investigators to use the Great Observatories, it is reasonable to expect similar treatment if investigators from the United States apply for observing time at foreign facilities. When searching for international partners, NASA should encourage potential foreign collaborators to establish such a spirit of reciprocity.

Although the details will differ from case to case, we believe that there are common principles that should guide the international development of facilities for space astrophysics. These include the following:

1. International cooperation should be nurtured by all available means in order to produce the best and most timely science and as a mechanism for reducing costs.

2. The best scientific programs result from planning by the scientists themselves. Measures should be taken to establish the communication channels to help ensure that scientific program coordination and/or cooperation occurs at the international level. 
3. The funding agencies should make every effort to coordinate their decision-making machinery so that the international partners can work effectively together. We note that a major problem in this respect is the apparent inability of NASA to maintain commitments or to adjust its apparatus to the procurement systems of other space agencies.

4. The management and operations of the international facilities should promote scientific exchange rather than simple mechanical divisions of the program tasks and objects of data analysis.

5. Scientific investigations should be supported on the basis of merit independent of the nationality of the proposers.

6. Meaningful contributions to operations and maintenance must be incorporated into international participatory agreements.

In summary we reach recommendation number 7 of our report:

7. We recommend that international cooperation be encouraged in all aspects of the long-lived observatory program. This cooperation should enhance the scientific productivity of the observatories and help reduce costs to NASA. Full participation of foreign partners in the sharing of the observatory maintenance and operations costs must be encouraged.

\section{GROUND-BASED OBSERVING SUPPORT}

Adequate ground-based observing support for astronomical observations from space requires (1) the existence of the necessary ground-based telescopes and instruments; (2) a mechanism for the common review of proposals that request time from both ground and space observatories; (3) a mechanism for scheduling the ground and space observations so that the relative times of the two sets of observations can meet the needs of the proposed scientific program; and (4) a strategy for the permanent storage and distribution of ground-based data. All of these requirements have been considered by the National Optical Astronomy Observatories (NOAO) Advisory Committee in their (1985) report to the director of NOAO on Coordination of Space and Ground-Based Observing. Below we briefly discuss and comment on the conclusions of the NOAO study. However, we have expanded our discussion and conclusions to include the important roles to be played by supporting ground-based studies in the radio region of the spectrum through the National Radio Astronomy Observatory. 
Many of our national ground-based observing facilities are heavily oversubscribed. This will create major scheduling problems in trying to coordinate ground- and space-based observing programs. To meet the expected demand, the NOAO study committee recommended the modification of existing facilities and the construction of additional optical telescopes and instruments. The list of facility recommendations is extensive. We support the basic idea of modifying and expanding the national optical facilities to make telescope time more readily available. However, decisions relating to the appropriate sizes, numbers, and location of new facilities are complex and must be considered within the broad context of the entire national program of astronomy and astrophyics.

The proposal review mechanism suggested by the NOAO Advisory Committee for programs common between NOAO and HST suggests that the meeting times of the two Telescope Allocation Committees (TACs) be phased to facilitate common reviews. The proposer would submit both the space- and ground-based portions of his other proposal to both the NOAO and HST TACs. The NOAO TAC would pass along its judgment of the proposal to the HST TAC, prior to its meeting, so that this TAC would know that "provisional" ground-based time had been granted. One or more common members of the HST and NOAO TACs would facilitate negotiation between these committees on common proposals. More detailed procedures for implementing programs requiring both space- and ground-based observing time are being considered by the Space Telescope Science Institute. Similar procedures could presumably be established to assign observing time to programs on various other ground-based observatories and future space facilities such as GRO, AXAF, and SIRTF. However, as the number of facilities increases, it will become increasingly difficult to coordinate the allocation cycles. A major concern pertaining to the coordination of ground and space observing programs is the double jeopardy character of the request procedure, that is, both the ground-based and the space-based proposals must be accepted for the program to be viable. With two allocation committees involved, the chance of success of such a program will certainly be less than for a proposal involving a single facility. Both TACs should be aware of this barrier to scientifically productive coordinated programs.

Coordination in the scheduling of space- and ground-based 
programs is another problem area. While scheduling of both types of observations must satisfy a number of constraints, those inherent for the space-based observatory are usually closely linked with the remainder of the schedule unit's programs rather than with controllable factors such as lunar phase and seasonal weather. In addition, instrument changes occur at much longer time intervals at most ground-based telescopes.

Schedule coordination must be an interactive process with something resembling the following sequence:

- ground-based observatory identifies windows acceptable for each proposal receiving a time allocation within a quarter or semester;

- space-based scheduler loads these constraints into the longterm plan and specifies the dates and times the telescope will be used for the given observations;

- ground-based facility builds a master schedule around these specific epochs.

A study of the feasibility of blocking out specific observations up to 9 months in advance to act as basic constraints to the spacecraft scheduler should be undertaken. Coordination of observations between two space-based facilities should also be considered at an early phase of mission planning-orbits $180^{\circ}$ out of phase for HST and AXAF would, for example, clearly exclude any possibility of most potentially fruitful simultaneous observing programs.

The NOAO Advisory Committee also recognized in its recent report that the National Optical Astronomy Observatories must soon develop reliable schemes for archiving ground-based astronomical data. A great deal of valuable data has already or will soon be lost to future generations of astronomers because of inadequate attention to this very important aspect of scientific research. As part of an archiving program, an explicit policy on proprietary rights to ground-based astronomical data must be established. The space-based data archives produced by such satellites as IUE, Einstein, and IRAS have proven to be international scientific treasures. The National Optical and Radio Astronomy Observatories should move rapidly to establish similarly valuable archives of their ground-based data. Rules on proprietary rights and archive 
TABLE 1 Operations and Maintenance Costs

\begin{tabular}{ll} 
Mission & Estimated Yearly Operations and Maintenance Costs (1986 M\$) \\
\hline HST & 160 \\
GRO & 20 (approximately) \\
AXAF & $50-60$ (Phase B estimate) \\
SIRTF & $\begin{array}{l}\text { not yet studied in detail, but probably similar to those for } \\
\end{array}$ \\
& AXAF
\end{tabular}

access should be made in coordination with such policies on spacebased facilities to minimize bureaucratic impediments to multiwavelength archival studies. Funding of such studies should also receive attention; the recent NASA decision to pool resources for its numerous guest investigator programs in order to allow scientific considerations to allocate the available resources is a good example to examine in the search for a coordinated policy.

These arguments lead to the eighth recommendation in the summary:

8. We recommend that the astronomical community establish and implement detailed plans for providing complementary and supporting ground-based observations to the measurements expected from the Great Observatories.

\section{COST CONTROL}

The annual cost to support HST activities is expected to approach $\$ 160$ million (1986\$). These costs will be shared approximately equally between the operational overhead (including spacecraft and the instrument control activities, STScI, the General Observer program), and the servicing charges (for example, maintenance, new instrument development). This annual support requirement for HST is both a necessary and sufficient reason to be concerned about the analogous costs for future observatories. It is instructive, therefore, to attempt to analyze these costs in comparison with what might be expected for AXAF and SIRTF. As noted in Table 1 (below) the estimated costs for GRO are so low in comparison with those for HST that they are not cost drivers in the Great Observatory program. 
In comparing the planned activities for HST, AXAF, and SIRTF, it is recognized that the HST flight operations are themselves quite complex. This complexity can be traced, ultimately, to the necessity that HST provide a data base that will significantly enhance the quality of data in a wavelength band that overlaps with ground-based research and that will support a much larger user community than anticipated for AXAF or SIRTF. Both AXAF and SIRTF operate in wavebands that are less deeply explored, and therefore do not require the $<0.01$ arcsecond pointing capability of HST, or its milliarcsecond aspect reconstruction (AXAF and SIRTF requirements are more than an order of magnitude less severe). Other nontrivial flight operations complexities of HST that are not shared by AXAF and SIRTF are the necessity for real-time operations, the simultaneous operation of two focal plane instruments, and the multiplicity of operational modes for each instrument. AXAF and SIRTF both have instruments that typically operate in "standard" operational modes, and both spacecraft utilize a single focal plane instrument in only preplanned observing sequences. In addition, other operational requirements for AXAF and SIRTF are significantly less burdensome than for HST. (The AXAF telemetry rate is more than an order of magnitude smaller, for example.)

In terms of operational complexity, perhaps the most important distinction between HST and other Great Observatories, is that HST does not have a true "safe" mode. As a result, immediate reaction to failures is needed to ensure that further (and perhaps fatal) failures do not occur. AXAF, by contrast, can survive extended periods of time with no ground commands through passive thermal control and a totally autonomous safe mode. As a consequence of this fundamental design difference, the size requirements for operations centers, the degree of contingency planning and support logistics, and the complexity of on-board systems for AXAF are all reduced relative to HST. The phase- $B$ cost estimates for AXAF in the areas of maintenance, and repair, and replacement instruments are only one third those for HST, reflecting in concrete terms the differences in these programs. To some extent these savings result from using the HST experience (that is, learning from the past) and a better appreciation of the real costs of Space Shuttle/Space Station services. As mentioned above, sensitivity to the high cost of operations (brought about by the HST estimates) has imposed a discipline on the AXAF 
and SIRTF programs to design in simplicity, reliability, and less demanding requirements in order to control operating costs.

There are also expected to be reductions in the cost for implementing the operations and data distribution and analysis activities, arising in part as a result of lessons learned in the HST program. As pointed out in the 1986 Astrophysics Data Operations report, the early identification of responsible individuals and organizations should prevent a large fraction of the inefficiencies that were manifested in the HST program: in revisions of specifications ("post-facto upgrades to the hardware and software systems have been expensive to implement..."), multiple contractors ("...the HST ground software system is large, complex and must be maintained and operated for many years by persons other than the developers... This is inherently an expensive process..."), and unworkable management interfaces ( $"$.... the ground system was developed in response to diverse and sometimes conflicting pressure, with attendant escalation in complexity.") The report goes on to add: "Many of these factors affecting ground system complexity are unique to the HST project and need not be of concern to other future missions." Also, as pointed out in that study, it is essential to avoid the fractionated program management with several NASA centers, that burdened the HST program. Other aspects of the HST operational overhead that have large ( $>\$ 10$ million/year) specific associated charges are the annual support of the Space Telescope Science Institute and the General Observer program. The subject of institutional arrangements for SIRTF and AXAF is covered in a separate section; we note here only that we do not recommend new institutional entities of size comparable to the Space Telescope Science Institute for those missions. The General Observer program for the HST has been scoped to accommodate the support requirements for the entire space astronomy community, so that we anticipate increases in the overall funding of space astronomy. In the case of AXAF and SIRTF, the user community is somewhat smaller and they are likely to be familiar with satellite observations. The latter assertion stems from there having been previous missions in these disciplines, HEAO (Einstein) and IRAS. Again, using the phase-B cost estimates for AXAF as a basis for comparison, there is a difference of about a factor of two in costs relative to HST estimates.

The space servicing segment of the operations costs is not quite as easily analyzed because, unlike the ground segment, the 
corresponding HST comparison system does not yet exist. Further, it is not clear what assumptions can be made about the availability of the Space Shuttle, the Space Station, orbiting platforms, or orbiting transfer/maneuvering vehicles. We can be sure, however, that some baseline space servicing capability will have been instituted for HST in advance of AXAF and SIRTF, and that operational costs can be shared with HST (as the major fraction of the HST servicing appears to be associated with the standby overhead). A specific example might be the Shuttle carriers used to bring repair or replacement equipment from the ground to orbit. These could be shared among all of the Great Observatories.

Current AXAF Phase-B studies indicate servicing at 3-year intervals that would primarily involve replenishment of expendables and replacement of "black box" instruments. SIRTF is anticipated to have similar requirements. The Solar Maximum Mission repair has already demonstrated the possibility of at least lower-level "black box" instrument replacement with Space Shuttle EVA, and NASA has committed to a first Space Shuttle test of cryogen transfer in orbit. Servicing stategies for AXAF and SIRTF should be studied carefully as the NASA space transportation system is revitalized. Major cost savings in the servicing area might be possible if both AXAF and SIRTF are flown on orbiting platforms of similar design. Or at the very least, there may be a number of common elements for these missions. Studies carried out by the Marshall Space Flight Center and the AXAF phase-B contractors indicate that the AXAF and SIRTF mission requirements are similar in many areas and could be met with common components. However, without detailed information it is not possible to be more specific about the actual impact of cost controls in the space servicing operations at present.

The other major contributor to the servicing charges is the development of new instrumentation for focal plane replacement. To help control costs in this area, we recommend establishing an oversight committee with the authority to recommend priorities among servicing possibilities for the combination of HST, AXAF, and SIRTF. Such a committee could provide enough discipline in the generation of requirements that a fixed annual budget for the servicing of all three might be a workable arrangement. The cost for new replacement instrumentation would then have to be evaluated in comparison with the cost for such instrumentation for other observatories, other servicing requirements, and other costs. 
Similarly, pressure for "new starts" for replacement instrumentation would require a harder look at potential facility closedown scenarios than might otherwise occur. It is also worth noting, however, that at least a portion of the HST, AXAF, and SIRTF instrumentation is already approaching its asymptotic capability, in the sense that neither photometers with efficiencies close to unity nor imagers with spatial resolution that is better than the facility optics need be improved.

In summary, there appears to be the opportunity for responsible cost control in the post-HST space observatories. With "data" from the history of HST ground operations, it can be anticipated that the current AXAF and SIRTF requirements will lead to significantly reduced costs compared to those for HST. Space servicing presents a more difficult activity to model in terms of absolute numbers because of uncertainties in future NASA baseline capabilities and their costs. We can demonstrate, however, that the AXAF and SIRTF costs will not require resources that are integral multiples of those for HST and, further, that we think it appropriate for the program of space observatories to have priorities established by its scientific community for the totality of available resources, in order that significant new operational expenditures be effectively balanced by forced reductions in other aspects of the operational space astrophysics program. In Table 1, we display a summary of estimated operations and maintenance costs of the different projects in 1986 dollars.

\section{RECOMMENDATIONS FOR THE MANAGEMENT OF THE SCIENCE OPERATIONS CENTER}

In the previous sections we have presented the major considerations relating to the science operations of future space observatories. Now we address the issue, based on the foregoing considerations, of what is the preferred institutional arrangement for a science operations center associated with a particular observatory. We agree with the conclusion in the Hornig report that, in view of the very complex scientific operations associated with any major space observatory, scientific management should be undertaken by an organization dedicated to this purpose; we have referred to this organization as the Science Operations Center (SOC). We also agree with the Martin report that it is essential that at least the core staff be created early in the program before engineering 
decisions are made without a full understanding of their ultimate implications. However, we have also recognized that with modern telecommunications capabilities, the Science Operations Center can be distributed; that is, it could consist of a small central operations center that has overall responsibility for coordinating operations and providing liaison with the user community and with NASA, and that is linked to Instrument Operations Centers that could be located at other institutions.

Important tasks for the Science Operations Center include the following:

1. Coordination of the scientific program.

2. Monitoring the scientific performance of the instruments and facility.

3. Interface with Guest Observers who use the observatory.

4. Development of the Observing Program.

5. Scheduling of observing time.

6. After launch, verification of instrument performance through quick-look science data.

7. Coordination of the Guest Observer proposals through the peer review process.

8. Coordination of special observations.

9. Support to the planning and execution of maintenance and refurbishment operations.

10. Assistance in definition and development of replacement instruments.

11. Coordination of software development standards.

12. Coordination of activities with other astrophysical observatories.

13. Coordination or consultation in communication of the achievements and importance of the observatory to both the scientific community and the public at large.

For the Space Telescope, the Hornig Committee recommended, and NASA concurred, that an independent scientific institute, the Space Telescope Science Institute, be formed to provide this scientific leadership. Although this appears to be working for the Hubble Space Telescope, we have considered several other modes for providing the scientific management framework for future observatories that operate at different wavelengths and have different operating requirements. Several options that we have considered are as follows: 
1. Use of the existing Space Telescope Science Institute for future space observatories.

2. Creation of a new Institute associated with each new observatory

3. Incorporation of the SOC into a NASA center.

4. Incorporaton of the SOC into an existing universityaffiliated astronomical research center.

5. Adoption of a distributed SOC. In this case there would be a small, central operations center that would meet most of the tasks outlined above, but the Instrument Operations Centers could be located at different sites.

After reviewing the operations of several existing operations centers, including IRAS, IUE, and STScI, and discussing the various approaches with members of the astronomical community, we believe that any of the above approaches can work to produce outstanding scientific results. Given the rapid developments in computing power and computer links, distributed scientific operations may play a stronger role in the future than in the past. The specific approach should be determined from the unique requirements of each mission, and the implementation should be determined by a competitive procedure that allows different approaches to be proposed.

\section{APPLICATION TO OTHER MISSIONS}

Many of the problems we have addressed are not unique to the Astrophysical Observatories that are the subject of this report. We have identified needs for an extensive data analysis capability, a dedicated support staff, and stable funding for investigator teams. These needs are not driven by the specific nature of the observatories in question-HST, AXAF, SIRTF, and GRO-but rather by the complexity of the scientific investigations envisioned and by the enhanced capability of modern instrument systems to provide large quantities of high-quality measurements. Indeed, these same problems are all being felt at ground-based optical and radio observatories where analogous requirements are found for the analysis of astronomical images and VLA data. By recognizing the community of problems and experimental requirements, a sharing of effort and reduction of overall cost becomes possible. An important example of a shared development is the Image Reduction and Analysis Facility (IRAF) software systems, which is 
a joint project of the STScI and the National Optical Astronomy Observatories.

The issues that we have considered in connection with longlived space observatories also arise for long-duration missions in other fields of space science. Questions of operations planning, distribution of large quantities of data, and support of a vigorous scientific research program are equally applicable to long-duration planetary, solar, and earth-observation missions. By developing effective strategies for efficiently and economically carrying out such missions, NASA can maximize the scientific return of its entire space science program. 


\section{Appendix: \\ Abbreviations Used in Text}

$\begin{array}{ll}\text { AXAF } & \begin{array}{l}\text { Advanced X-Ray Astrophysics Facility } \\ \text { COmmittee on Data Management and Coordi- } \\ \text { nation }\end{array} \\ \text { EUVE } & \begin{array}{l}\text { Extreme Ultraviolet Explorer } \\ \text { GRO }\end{array} \\ \text { Gamma Ray Observatory } \\ \text { HEAO } & \text { High Energy Astrophysical Observatory } \\ \text { HST } & \text { Hubble Space Telescope } \\ \text { IRAS } & \text { Infrared Astronomical Satellite } \\ \text { ISO } & \text { Infrared Space Observatory } \\ \text { IUE } & \text { International Ultraviolet Explorer } \\ \text { NASA } & \text { National Aeronautics and Space Administration } \\ \text { NASCOM } & \text { NASA Communications } \\ \text { NOAO } & \text { National Optical Astronomy Observatories } \\ \text { OMV } & \text { Orbital Maneuvering Vehicle } \\ \text { PI } & \text { Principal Investigator } \\ \text { ROSAT } & \text { Roentgen Satellite } \\ \text { SIRTF } & \text { Space Infrared Telescope Facility } \\ \text { SOC } & \text { Science Operations Center } \\ \text { STScI } & \text { Space Telescope Science Institute } \\ \text { TAC } & \text { Time Allocation Committee } \\ \text { TDRSS } & \text { Tracking and Data Relay Satellite System } \\ \text { VLA } & \text { Very Large Array }\end{array}$


